\title{
Nonsingular Fast Terminal Sliding Mode Control with Extended State Observer and Tracking Differentiator for Uncertain Nonlinear Systems
}

\author{
Zhenxin He, Chuntong Liu, Ying Zhan, Hongcai Li, Xianxiang Huang, and Zhili Zhang \\ Xian Research Institute of High Technology, Baqiao District, Tongxin Road, Xi'an City, Shaanxi 710025, China \\ Correspondence should be addressed to Chuntong Liu; liuchuntong2001@163.com
}

Received 7 June 2014; Revised 14 September 2014; Accepted 29 September 2014; Published 27 October 2014

Academic Editor: Shihua Li

Copyright (C) 2014 Zhenxin He et al. This is an open access article distributed under the Creative Commons Attribution License, which permits unrestricted use, distribution, and reproduction in any medium, provided the original work is properly cited.

A continuous nonsingular fast terminal sliding mode (NFTSM) control scheme with the extended state observer (ESO) and the tracking differentiator (TD) is proposed for second-order uncertain SISO nonlinear systems. The system's disturbances and states can be estimated by introducing the ESO, then the disturbances are compensated effectively, and the ideal transient process of the system can be arranged based on TD to provide the target tracking signal and its high-order derivatives. The proposed controller obtains finite-time convergence property and keeps good robustness of sliding mode control (SMC) for disturbances. Moreover, compared with conventional SMC, the proposed control law is continuous and no chattering phenomenon exists. The property of system stability is guaranteed by Lyapunov stability theory. The simulation results show that the proposed method can be employed to shorten the system reaching time, improve the system tracking precision, and suppress the system chattering and the input noise. The proposed control method is finally applied for the rotating control problem of theodolite servo system.

\section{Introduction}

Sliding mode control (SMC) is well known for its robustness to system parameter variations and external disturbances [1]. SMC has been widely used for robots, aircrafts, DC and AC motors, power systems, process control, and so on $[2,3]$. In general, when the linear sliding mode surface function is chosen, the tracking errors of the system states will not be converged to zero within finite time. So, the nonlinear item is used to obtain the better performance response in the sliding mode surface [4]. Based on the terminal attractor, Zhihong et al. proposed TSM control method to offer finite time convergence property $[5,6]$. Following it, many improved forms of TSM are studied. There is a singularity problem in the existing TSM controller design methods [7]. So, Feng et al. proposed a NTSM control method to overcome the singular problem [8]. In [9], Yu and Zhihong developed fast terminal sliding mode control (FTSM). Then, a nonsingular fast terminal sliding mode was proposed to overcome the controller singular problem and the fast convergence problem [10].
Chattering is an unavoidable problem for the SMC technology in the actual application including above NTSM control schemes. So some effective methods are proposed [11-13]. The chattering phenomenon is avoided. However, to do this, the disturbance rejection properties are sacrificed to some extent. Yang et al. proposed a new continuous dynamic sliding mode control (CDSMC) method for high-order mismatched disturbance attenuation in motion control systems [14]. To completely compensate the effects of disturbances, the high-order sliding mode differentiator is used to estimate the mismatched high-order disturbance and its high-order derivatives.

It is difficult to design the appropriate sliding mode gain $k$ for the time-varying system disturbance as the main factor of chattering. Furthermore, it is usually impossible to measure the disturbances directly in some certain applications, for example, PMSM control system. Ohnishi presented disturbance observer (DOB) technique firstly [15]. Based on DOB technique, Wei et al. firstly proposed many types of DOB-based SMC methods to improve the system performance [16-18]. Reference [19] proposed a nonlinear 
disturbance observer to estimate and compensate the external disturbances. The switching gain was reduced greatly, and the chattering was avoided effectively. Yang et al. proposed a continuous nonsingular terminal sliding mode control approach with DOB for mismatched disturbance attenuation [20]. Su et al. designed a finite-time disturbance observer (FTDO) to estimate the uncertainties for robotic manipulators tracking control [21].

However, except for the system disturbance, we may be interested in some interior states of the system which cannot be all measured directly by sensors. It will waste hardware too much and gain the dissatisfactory results even though some of them can be obtained in the control practice. For example, in the SISO motor motion system, the velocity signal is usually obtained by using many kinds of encoders. Encoders measure the position signal directly to get the velocity signal by adopting different method. But the measuring precision and antijamming problem need to be studied furthermore. Encoders enlarge the system size and increase the fees. In addition, the high-order derivatives of reference position signal with other noise signals need to be usually provided for the single input motion system based on SMC method. Extended state observer (ESO) and tracking differentiator (TD) of the active disturbance rejection control (ADRC) originated from Professor Han offer good and novel thought to solve the above problems [22]. It is not necessary to measure the accurate system states and the disturbance, while ESO will estimate them in real time. TD is used to obtain the differential signal mixed with other noise signal via integration quickly and accurately. Gao, Huang and Xue, and Xia et al. have given ADRC application and popularization and ADRC connotation summary [23-25].

In this paper, an improved continuous nonsingular fast terminal sliding mode (NFTSM) control is proposed for a class of uncertain nonlinear system involving external disturbances and input reference signal with the noise signal. The main contributions of this paper are as follows. First, the finite-time convergence property can be guaranteed even in the presence of disturbances. An improved fast terminal sliding mode surface function with a novel fast reaching law is designed to improve the convergence rate along the sliding surface. The reaching law is designed to shorten the reaching time and enhance the design flexibility. Second, the nominal performance is retained with the proposed method as the modules of the controller are independent of each other; that is, the proposed method acts the same as the regular SMC in the absence of disturbances. Third, the proposed controller is continuous without any chattering since the disturbances are due to ESO, which does not lose the robustness property and the precision, and the sliding mode switching item of the controller is removed for disturbance rejection. Fourth, except for disturbances, ESO is also used to estimate the system states. It will avoid measuring certain interior states of the system directly by sensors, which wastes hardware too much and gains the dissatisfactory results even though some of them can be obtained in the control practice. TD is introduced to obtain the reference input signal and its high-order derivatives, and it is very suitable for SMC and can extract the useful and precise signal from the noise. So,
ADRC and NFTSM control are successfully integrated with uncertain nonlinear systems firstly in this paper.

Theodolite is widely used in the fields of geodetic surveying, weapon system, and engineering surveying [26]. Theodolite rotating control is put forward taking shaft motor drive as the core to improve the theodolite measure precision and automation degree by combining theodolite with servo drive technology [27]. However, essentially, theodolite rotating system is a nonlinear system subject to both external disturbances and parameter variations. Recently, its control methods mainly include improved PID control [28] and adaptive control [29].

The rest of the paper is organized as follows. In Section 2, the background review of NTSM, TD, and ESO is outlined. In Section 3, the proposed nonsingular fast terminal sliding mode controller with $\mathrm{ESO}, \mathrm{TD}$, and fast reaching law is derived and the stability of the control system is proved by Lyapunov's stability theory. Simulation results are presented in Section 4, and the method proposed is applied to theodolite rotating control in this section. Finally, conclusions are provided in Section 5.

\section{Background Review}

2.1. NTSM. Consider the following second-order SISO system with nonlinear uncertainties described by

$$
\begin{aligned}
& \dot{x}_{1}=x_{2}, \\
& \dot{x}_{2}=f(x)+b(x) u(x)+g(x, t), \\
& y=x_{1},
\end{aligned}
$$

where $x=\left[x_{1}, x_{2}\right]^{T} \in R^{2}, u \in R$, and $y \in R$ are the states variables, the control input, and the output of the system, respectively; both $f(x)$ and $b(x) \neq 0$ are the smooth nonlinear function in terms of $x ; g(x, t)$ is the uncertainty and disturbance satisfying $|g(x, t)|<l_{g}$, and $l_{g}>0$.

The conventional TSM is described by the following surface function [5]:

$$
s=e_{2}+\beta e_{1}^{q / p},
$$

where $e_{1}=x_{1}-x_{d}$ is the tracking error and $e_{2}=\dot{x}_{1}-\dot{x}_{d}$, as $x_{d}$ is the reference signal. $\beta>0$ is the designed parameter, and $p$ and $q$ are the positive odd integers satisfying $q<p<2 q$.

For system (1), a TSM controller is designed as follows:

$$
u=-b^{-1}(x)\left[f(x)+\beta \frac{q}{p} e_{1}^{q / p-1} e_{2}+\left(l_{g}+\eta\right) \operatorname{sgn}(s)-\ddot{x}_{d}\right],
$$

where $\eta>0$ is a constant. The controller can ensure that the TSM occurs. Analyzing the TSM surface function (2), we can know that the system states errors $e_{1}$ and $e_{2}$ converge to zero in the finite time.

It can be seen in the TSM controller (3) that the second term containing $e_{1}^{q / p-1} e_{2}$ may cause a singularity if $e_{2} \neq 0$ and $e_{1}=0$. This situation does not occur in the ideal sliding mode when $s=0, e_{2}=-\beta e_{1}^{q / p}$. Substitute $e_{2}=-\beta e_{1}^{q / p}$ into $e_{1}^{q / p-1} e_{2}$ 
to get $e_{1}^{(2 q-p) / p}$. If $q<p<2 q, e_{1}^{(2 q-p) / p}$ is nonsingular. The singularity problem may occur in the reaching phase when there is an insufficient control to ensure that $e_{2} \neq 0$ and $e_{1}=0$ [8]. TSM controller (3) cannot guarantee a bounded control signal for the case of $e_{2} \neq 0$ when $e_{1}=0$ before the system states reach $s=0$. Furthermore, the singularity may also occur even after the sliding mode $s=0$ is reached since, due to computation errors and uncertain factors, the system states cannot be guaranteed to always remain in the sliding mode especially near the equilibrium point $\left(e_{1}=0 ; e_{2}=0\right)$, and the case of $e_{2} \neq 0$ while $e_{1}=0$ may occur from time to time. This underlines the importance of addressing singularity problem in the TSM systems.

To solve the controller singularity problem and improve the system tracking precision, the NTSM control is presented. For (1), NTSM surface function is described as follows [8]:

$$
s=e_{1}+\frac{1}{\beta} e_{2}^{p / q},
$$

where $\beta$, $p$, and $q$ have been defined in (2). For system (1) with NTSM (4), the controller can be designed as follows:

$$
u=-b^{-1}(x)\left[f(x)+\beta \frac{q}{p} e_{2}^{2-p / q}+\left(l_{g}+\eta\right) \operatorname{sgn}(s)-\ddot{x}_{d}\right],
$$

where $1<p / q<2 ; \eta>0$ then the NTSM surface function (4) will be reached in the finite time. Furthermore, the states errors $e_{1}$ and $e_{2}$ will converge to zero in the finite time. It should be noted that the NTSM controller (5) is always nonsingular in the state space since $1<p / q<2$. However, the system states reach the sliding mode $s=0$; it is obtained as follows:

$$
\dot{e}_{1}=-\left(\frac{1}{\beta}\right)^{q / p} e_{1}^{q / p}
$$

Remark 1. The system states can reach the sliding mode surface $s=0$ by designing the reasonable sliding mode controller to satisfy Lyapunov stability principle. Suppose that the reaching time is $t_{r}$. When the system reach the sliding mode surface, $e_{1}$ will decrease as $e_{1}>0$ due to $\dot{e}_{1}<0$ from (6); $e_{1}$ will increase as $e_{1}<0$. Finally, the system errors can converge to 0 in finite time $t_{s 1}$ by solving (6). However, the system states converge slowly for the regions far away from the equilibrium point because the exponent of $e_{1}$ is less than 1 according to (6).

Remark 2. For the NTSM controller (5), in order to achieve system convergence, the switching gain $\eta$ is generally selected for a bigger value. It will produce the chattering obviously and influence the convergence precision in the steady state, even the unmodeled dynamics of the system are motivated by the chattering to make the system unstable.

From above analysis, it is very necessary to improve system convergence characteristics and eliminate system chattering based on the conventional NTSM controller.
2.2. Tracking Differentiator. In the transition design of the reference signal, both transition signal and its derivatives are simultaneously presented. The differential signal is usually obtained by the backward difference of the reference signal, but it will get unstable and inaccurate results in the presence of noise. However, TD has the ability to resolve the problem of differential signal extraction via integration to avoid unnecessary noise and make the system performance more effective and robust in some situations [25]. A feasible second-order TD can be designed as follows:

$$
\begin{aligned}
& \dot{v}_{1}=v_{2}, \\
& \dot{v}_{2}=\text { fhan }\left(v_{1}-v(t), v_{2}, r, h_{0}\right),
\end{aligned}
$$

where $v(t)=x_{d}$ denotes the control objective. $v_{1}$ is the desired trajectory and $v_{2}$ is its derivative. $r$ and $h_{0}$ are the controller's parameters. $r$ is the speed factor deciding tracking speed. $h_{0}$ is the filtering factor, which makes an effort of filter. These parameters can be adjusted individually according to the desired speed and smoothness. fhan $\left(v_{1}-v(t), v_{2}, r, h_{0}\right)$ is defined as follows [19]:

$$
\begin{gathered}
d=r h_{0}^{2}, \quad a_{0}=h_{0} v_{2}, \quad y=\left(v_{1}-v(t)\right)+a_{0}, \\
a_{1}=\sqrt{d(d+8|y|)}, \\
a_{2}=a_{0}+\frac{\operatorname{sign}(y)\left(a_{1}-d\right)}{2}, \\
s_{y}=\frac{(\operatorname{sign}(y+d)-\operatorname{sign}(y-d))}{2}, \\
a=\left(a_{0}+y-a_{2}\right) s_{y}+a_{2}, \\
s_{a}=\frac{(\operatorname{sign}(a+d)-\operatorname{sign}(a-d))}{2}, \\
\text { fhan }=-r\left(\frac{a}{d}-\operatorname{sign}(a)\right) s_{a}-r \operatorname{sign}(a) .
\end{gathered}
$$

TD with nonlinear feedback combination can provide transition process for expected input $v=x_{d}$, that is, $v_{1}$ and its differential $v_{2}$. Meanwhile, TD has the ability to track input reference signal with quick response and avoid overshooting.

2.3. Extended State Observer. In the theoretical analysis, it is assumed that the estimation error caused by using ESO is rather small. This fact is coupled severely with the desirable result: the states are stable. Otherwise, the disturbance may be unbounded and that the estimation error is meaningless.

$w(x, t)=f(x)+g(x, t)$ is a multivariable function of the states, external disturbances, and time. Generally, it is difficult to get the exact model of $w(x, t)$. Now, the ESO is used to estimate $w(x, t)$ in real time and make adjustments at each sampling point in a digital controller. We define $x_{3}$ as the 
derivative of the uncertainties. The original system (1) can be rewritten as follows:

$$
\begin{aligned}
& \dot{x}_{1}=x_{2}, \\
& \dot{x}_{2}=x_{3}+b u, \\
& \dot{x}_{3}=a(t), \\
& x_{1}=y,
\end{aligned}
$$

which is always observable. Now, we construct a state observer to estimate $x$ and $w(x, t)$, in the following form:

$$
\begin{aligned}
e & =z_{1}-y \\
\dot{z}_{1} & =z_{2}-\beta_{1} e \\
\dot{z}_{2} & =z_{3}-\beta_{2} \mathrm{fal}\left(e, \alpha_{1}, \delta\right)+b_{0} u, \\
\dot{z}_{3} & =-\beta_{3} \mathrm{fal}\left(e, \alpha_{2}, \delta\right),
\end{aligned}
$$

where $z_{1}, z_{2}$, and $z_{3}$ are the observer outputs and $\beta_{1}, \beta_{2}$, and $\beta_{3}$ are the observer gains. $e$ is the output error of the system. $z_{1}$ is used to estimate $x_{1} ; z_{2}$ is used to estimate $x_{2} ; z_{3}$ is the extended state variable to estimate the comprehensive disturbance $w(x, t)$. As to a particular problem, the observer gains, $\beta_{1}, \beta_{2}$, and $\beta_{3}$, must be selected rationally to guarantee the speed and precision of the observer [30]. The function of $\mathrm{fal}(\cdot, \cdot, \cdot)$ is defined as

$$
\mathrm{fal}(e, \alpha, \delta)= \begin{cases}|e|^{\alpha} \operatorname{sgn}(e), & |e|>\delta \\ \frac{e}{\delta^{1-\alpha}}, & |e|<\delta\end{cases}
$$

where $\delta>0$ and $0<\alpha_{2}<\alpha_{1}<1$ are parameters that can be regulated.

Let $\varepsilon_{1}(t)=z_{1}(t)-x_{1}(t), \varepsilon_{2}(t)=z_{2}(t)-x_{2}(t)$, and $\varepsilon_{3}(t)=$ $z_{3}(t)-x_{3}(t)$. From (9) and (10), we have

$$
\begin{aligned}
& \dot{\varepsilon}_{1}(t)=\varepsilon_{2}(t)-\beta_{1} \varepsilon_{1}(t), \\
& \dot{\varepsilon}_{2}(t)=\varepsilon_{3}(t)-\beta_{2} \text { fal }\left(\varepsilon_{1}(t), \alpha_{1}, \delta\right), \\
& \dot{\varepsilon}_{3}(t)=-\beta_{3} \text { fal }\left(\varepsilon_{1}(t), \alpha_{2}, \delta\right)-h(t),
\end{aligned}
$$

with the following constraints on the observer parameters

$$
\begin{array}{r}
\varepsilon_{1}^{2} \beta_{1}>0, \quad \varepsilon_{1} \mathrm{fal}\left(\varepsilon_{1}, \alpha_{1}, \delta\right)>0, \quad \varepsilon_{1} \mathrm{fal}\left(\varepsilon_{1}, \alpha_{2}, \delta\right)>0, \\
\forall \varepsilon_{1} \neq 0, \text { fal }\left(0, \alpha_{1}, \delta\right)=0 .
\end{array}
$$

It is shown in [31] that for an arbitrarily changing $h(t)$ in a certain range, the aforementioned system is stable with respect to the origin. This means, with appropriate choices of parameters $\alpha_{1}, \alpha_{2}, \beta_{1}, \beta_{2}, \beta_{3}$, and $\delta$, that the states of the system (10) can track the corresponding states of the system $(9)$; that is, $z_{1}(t) \rightarrow x_{1}(t), z_{2}(t) \rightarrow x_{2}(t)$, and $z_{3}(t) \rightarrow x_{3}(t)$. So the system (10) is called the extended state observer of the system (1) and is used to estimate $x_{i}(t), i=1,2,3$.

The system output $y(t)$ needs to track a reference input signal $v(t)=x_{d}$. The tracking differentiator is used to arrange the transient process $v_{1}(t)$ that tracks the reference input signal $v(t)$, and then the extended state observer uses the system output $y(t)$ as its input rather than $v_{1}(t)$ itself. In addition, the nonlinear state error feedback (NLSEF) is the ADRC control law. In order to improve the system control performance, a novel NFTSM controller will replace the traditional nonlinear PID controller in this study.

\section{The NFTSM Controller Design with ESO and TD}

In this section, the new NFTSM surface and the sliding mode reaching law are proposed. Then, the NFTSM controller is designed and some of its properties are analyzed. At last, the complete structure of the NFTSM controller with TD and ESO is given.

\subsection{A New Form of TSM Surface Function}

Definition 3. For (1), the NFTSM can be described by the following first-order nonlinear differential equation:

$$
s=e_{1}+k_{1}\left|e_{1}\right|^{\gamma+1}+k_{2} e_{2}^{p / q}
$$

where $k_{1}, k_{2}$, and $\gamma$ are all the designed constants; $k_{1}>0$, $k_{2}>0$, and $\gamma>0 ; p$ and $q$ are positive odd integers satisfying $1<p / q<2 . \gamma+1>p / q$. When the system states reach sliding mode surface $s=\dot{s}=0$, (14) can be described as follows:

$$
\begin{aligned}
\dot{e}_{1} & =-\left(\frac{1}{k_{2}}\right)^{q / p}\left(e_{1}+k_{1}\left|e_{1}\right|^{\gamma+1}\right)^{q / p} \\
& =-\left(\frac{1}{k_{2}}\right)^{q / p}\left(e_{1}+k_{1} e_{1}^{\gamma+1} \operatorname{sgn}\left(e_{1}\right)^{\gamma+1}\right)^{q / p} \\
& =-e_{1}^{q / p}\left(\left(\frac{1}{k_{2}}\right)\left(1+k_{1} e_{1}^{\gamma} \operatorname{sgn}\left(e_{1}\right)^{\gamma+1}\right)\right)^{q / p} .
\end{aligned}
$$

Suppose that the time from the initial state error $e(0) \neq 0$ to $e=0$ is $t_{s}$; that is, $e\left(t_{s}\right)=0$. The time integrate of (15) satisfies,

$$
\begin{aligned}
\int_{e_{1}(0)}^{e_{1}\left(t_{s}\right)} \frac{d e_{1}}{e_{1}^{q / p}} & =-\int_{0}^{t_{s}}\left(\frac{1}{k_{2}}\left(1+k_{1} e_{1}^{\gamma} \operatorname{sgn}\left(e_{1}\right)^{\gamma+1}\right)\right)^{q / p} d \tau \\
& \leq-\int_{0}^{t_{s}}\left(\frac{1}{k_{2}}\right)^{q / p} d \tau
\end{aligned}
$$

Furthermore,

$$
t_{s} \leq \frac{p}{\left(1 / k_{2}\right)^{q / p}(p-q)} e_{1}^{(1-q / p)}(0) .
$$

Remark 4. For the sliding mode surface (14), when the states are far from the equilibrium point, the first team $e_{1}$ of $s$ mainly affects the convergence efficiency, which can make the system trajectory converge quickly. When the states are near to the equilibrium point, the second team $k_{1}\left|e_{1}\right|^{\gamma+1}$ of $s$ largely affects the convergence efficiency, which can also make the 
system trajectory converge quickly. So the NFTSM scheme can achieve the global states trajectory converges quickly, not just within the area adjacent to the equilibrium point. In addition, the sliding mode surface (14) is slightly different from the previously reported FTSM which is expressed as [9]

$$
s=e_{2}+\alpha e_{1}+\beta e_{1}^{q / p}=0
$$

where $\alpha, \beta>0, p>q>0$ are integers and $p$ and $q$ are odd.

Except for the controller singularity, the difference of the sliding mode surface $s$ between the proposed NFTSM and the FTSM is their second teams affecting the near convergence area. The system (14) will show a better character than the system (18). The convergence time along the sliding mode surface $t_{f}$ can be obtained as follows [8]:

$$
t_{f}=\frac{1}{\alpha(1-q / p)} \ln \frac{\alpha\left|e_{0}\right|^{1-q / p}+\beta}{\beta},
$$

where $e_{0}=x_{1}(0)-x_{d}(0)$.

In [32], the existing nonsingular fast TSM control method is proposed, and the NFTSM surface function is described as follows:

$$
s=e_{1}+k_{1} e_{1}^{g / h}+k_{2} e_{2}^{p / q},
$$

where $k_{2} \in R^{+}, k_{2} \in R^{+}, p, q, g$, and $h$ are all odd and $1<$ $p / q<2, g / h>p / q$ to guarantee the system nonsingular characteristics.

According to [33], the convergence time along the sliding mode surface (20) $t_{\mathrm{nf}}$ can be obtained as follows:

$$
t_{\mathrm{nf}}=\int_{0}^{\left|e_{0}\right|} \frac{k_{2}^{q / p}}{\left(e_{1}+k_{1}\left|e_{1}\right|^{g / h}\right)^{q / p}} d e_{1},
$$

where $e_{0}=x_{1}(0)-x_{d}(0)$. The finite integration of (16) can be calculated by the gauss hypergeometric function.

Remark 5. (a) For the sliding mode surface (18), comparing with (14) and (20), the singularity phenomenon may occur in some area. If $e_{2} \neq 0$ and $e_{1} \neq 0$, the term $e_{1}^{q / p-1} e_{2} \notin$ $R$ in the controller, which leads to the singularity problem. The proposed surface function (14) does have this problem. Except for the controller singularity, the second teams of three sliding mode surfaces directly affect the near convergence area.

(b) Furthermore, the system (14) shows the global convergence property in the finite time. Analyzing (17), (19), and (21), the convergence time $t_{r}$ by using the proposed sliding mode surface is the smallest from the initial system states to the equilibrium point along $s=0$ when the designed parameters of the sliding mode functions are the same.

3.2. Design of the Reaching Law. Based on the sliding mode structure theory, the reaching condition of the sliding mode can just promise that the free point reaches the sliding mode surface in the finite time. But, the reaching specific trajectory

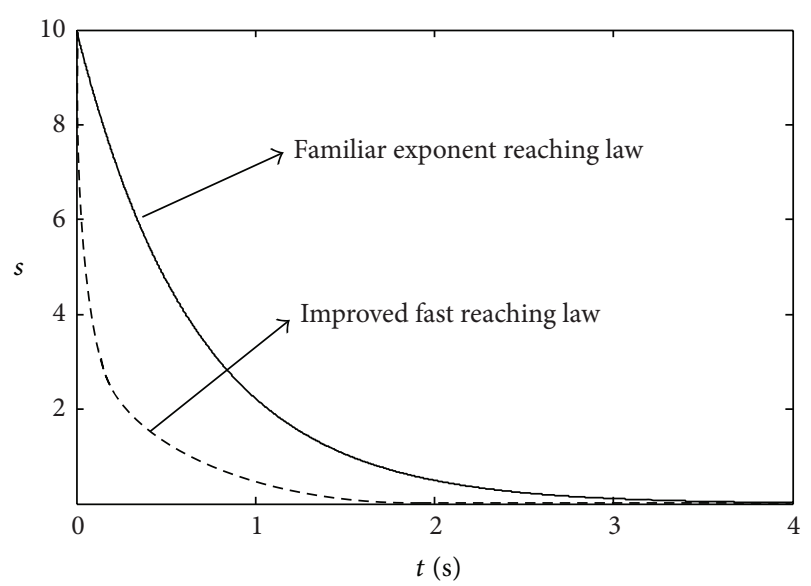

FIgURE 1: Performance comparison of reaching laws.

has not been limited, so the reaching law can improve the system dynamic quality of the reaching movement. The familiar exponent reaching law is given as follows [34]:

$$
\dot{s}=-\varepsilon \operatorname{sgn} s-k s, \quad \varepsilon>0, k>0,
$$

where $\dot{s}=-k s$ is the exponent team, and its solution is $s=$ $s(0) e^{-k t}$.

The exponent team can make the system states move to the sliding manifold. If there is only the exponent team, the reaching process is asymptotic, and it cannot achieve the reaching in finite time. So, the constant speed reaching team $\dot{s}=-\varepsilon \operatorname{sgn}(s)$ to promise the finite time reaching.

The familiar exponent reaching law can provide some references for the development of the reaching law.

In this paper, an improved fast reaching law is designed to shorten the reaching time and enhance the design flexibility. It is as follows:

$$
\dot{s}=-k_{3}|s|^{m} \operatorname{sgn}(s)-k_{4}|s|^{n / 2} \operatorname{sgn}(s),
$$

where $k_{3}>0$ and $k_{4}>0$ are the designed coefficients; the odd number $m>1$ and the rational number $2<n<4$ are the designed exponents. When the system is far away from the sliding mode surface, $-k_{3}|s|^{m} \operatorname{sgn}(s)$ plays the leading role to guarantee the faster reaching speed. When the system is near the sliding mode surface, $-k_{4}|s|^{n / 2} \operatorname{sgn}(s)$ plays the leading role to reduce the reaching speed that make the system states reach the sliding mode surface smoothly. Consider

$$
s \dot{s}=-k_{3}|s|^{m+1}-k_{4}|s|^{n / 2+1} \leq 0 .
$$

So, the improved fast reaching law satisfies the sliding mode reaching condition. Suppose that $\varepsilon=20, k=10$, $k_{3}=50$, and $k_{4}=50$; the performance of the improved reaching law and the familiar exponent reaching law is shown in Figure 1.

From Figure 1, the system reaching speed of proposed reaching law is bigger than that of exponent reaching law from the initial states to sliding mode surface. When the system is near the sliding mode surface, the reaching speed is down to reduce the initial chattering when the system enters the sliding mode surface. 


\subsection{Sliding Mode Controller Design and Stability Analysis}

Theorem 6. With the sliding mode surface function (14), the trajectory of system (1) can be driven onto the sliding surface with NFTSM controller (25), and finally evolves in a neighborhood around the origin in finite time:

$$
\begin{aligned}
u=-b(x)^{-1}\left[\widehat{\omega}(x, t)+k_{3}|\widehat{s}|^{m} \operatorname{sgn}(\widehat{s})+k_{4} \hat{s}^{n / 2}\right. \\
\left.\quad+\frac{1}{k_{2}} \widehat{e}_{2}^{2-p / q} \frac{q}{p}\left(1+k_{1}(\gamma+1)\left|\widehat{e}_{1}\right|^{\gamma}\right)-\ddot{x}_{d}\right],
\end{aligned}
$$

where $\widehat{e}_{1}=z_{1}-x_{d}, \widehat{e}_{2}=z_{2}-\dot{x}_{d} ; z_{1}$ and $z_{2}$ are the states estimations from ESO; $x_{d}, \dot{x}_{d}$, and $\ddot{x}_{d}$ are the reference signals from TD. Moreover, the sliding mode surface function can be described by $\widehat{s}=\widehat{e}_{1}+k_{1}\left|\widehat{e}_{1}\right|^{\gamma+1}+k_{2} \widehat{e}_{2}^{p / q}$ again. $\widehat{\omega}(x, t)=$ $\widehat{f}(x)+\hat{g}(x, t)$ is the estimation value of the comprehensive disturbances $\omega(x, t)=f(x)+g(x, t)$ from $z_{3}$ of ESO; $\mathbf{K}=$ $\left[k_{1}, k_{2}, k_{3}, k_{4}\right], m, n$, and $\gamma$ are positive design parameters; $p$ and $q$ are positive odd integers, and $1<p / q<2 ; m>1$ and $2>n>0$.

Proof. Consider the following Lyapunov function:

$$
V=\frac{1}{2} s^{2}
$$

Its time derivative is

$$
\begin{aligned}
& \dot{V}=s \dot{s}=s\left(e_{2}+k_{1}(\gamma+1)\left|e_{1}\right|^{\gamma} e_{2}+k_{2} \frac{p}{q} e_{2}^{p / q-1} \ddot{e}_{1}\right) \\
&=s\left(e_{2}+k_{1}(\gamma+1)\left|e_{1}\right|^{\gamma} e_{2}\right. \\
&\left.+k_{2} \frac{p}{q} e_{2}^{p / q-1}\left(\omega(x, t)+b(x) u-\ddot{x}_{d}\right)\right) .
\end{aligned}
$$

Combine (25) and (27) to yield

$$
\begin{gathered}
\dot{V}=s\left(e_{2}+k_{1}(\gamma+1)\left|e_{1}\right|^{\gamma} e_{2}+k_{2} \frac{p}{q} e_{2}^{p / q-1}\right. \\
\times\left(\omega(x, t)-\widehat{\omega}(x, t)-k_{3} \widehat{s}^{m}-k_{4} \widehat{s}^{n / 2}\right. \\
\left.\left.-\frac{1}{k_{2}} \widehat{e}_{2}^{2-p / q} \frac{q}{p}\left(1+k_{1}(\gamma+1)\left|\widehat{e}_{1}\right|^{\gamma}\right)\right)\right) \\
=k_{2} \frac{p}{q} e_{2}^{p / q-1} s\left[\frac{1}{k_{2}} \frac{q}{p}\left(e_{2}^{2-p / q}-\widehat{e}_{2}^{2-p / q}\right)\right. \\
+\frac{k_{1}(\gamma+1)}{k_{2}} \frac{q}{p}\left(\left|e_{1}\right|^{\gamma} e_{2}^{2-p / q}-\left|\widehat{e}_{1}\right|^{\gamma} \widehat{e}_{2}^{2-p / q}\right) \\
\left.-\widetilde{\omega}(x, t)-k_{3}(s-\widetilde{s})^{m}-k_{4}(s-\widetilde{s})^{n / 2}\right]
\end{gathered}
$$

where $\widetilde{\omega}(x, t)=\omega(x, t)-\widehat{\omega}(x, t)$.
Suppose $l\left(e_{2}\right)=k_{2}(p / q) e_{2}^{p / q-1}$, where $p$ and $q$ are positive odd integers; it should be satisfied that $1<p / q<2$. Hence when $e_{2} \neq 0, l\left(e_{2}\right)>0$. Due to $s \neq \widetilde{s} \neq 0, m$ is the odd number and $1<n / 2<2$, similarly, $s^{n / 2}>0$. According to the inequation theorem,

$$
\begin{gathered}
e_{2}^{2-p / q}-\widehat{e}_{2}^{2-p / q} \leq\left|\widetilde{e}_{2}\right|^{2-p / q} ; \\
\left|e_{1}\right|^{\gamma} e_{2}^{2-p / q}-\left|\widehat{e}_{1}\right|^{\gamma} \hat{e}_{2}^{2-p / q} \\
=\left|e_{1}\right|^{\gamma} e_{2}^{2-p / q}-\left|e_{1}-\widetilde{e}_{1}\right|^{\gamma} \widehat{e}_{2}^{2-p / q} \\
\leq\left|e_{1}\right|^{\gamma} e_{2}^{2-p / q}+\widetilde{e}_{1}^{\gamma} \widehat{e}_{2}^{2-p / q}-e_{1}^{\gamma} \widehat{e}_{2}^{2-p / q} \\
=\left|e_{1}\right|^{\gamma}\left(e_{2}^{2-p / q}-\widehat{e}_{2}^{2-p / q}\right)+\widetilde{e}_{1}^{\gamma} \widehat{e}_{2}^{2-p / q} \\
\leq\left|e_{1}\right|^{\gamma}\left|\widetilde{e}_{2}\right|^{2-p / q}+\widehat{e}_{2}^{2-p / q} \widetilde{e}_{1}^{\gamma} ; \\
(s-\widetilde{s})^{m} \geq s^{m}-\widetilde{s}^{m},
\end{gathered}
$$

where $\widetilde{s}=s-\widehat{s} ; \widetilde{e}_{1}=e_{1}-\widehat{e}_{1} ; \widetilde{e}_{2}=e_{2}-\widehat{e}_{2}$. Then

$$
\begin{aligned}
\dot{V} \leq l\left(e_{2}\right)[s( & \frac{1}{k_{2}} \frac{q}{p}\left|\widetilde{e}_{2}\right|^{2-p / q}+\frac{k_{1}(\gamma+1)}{k_{2}} \frac{q}{p} \\
& \times\left(\left|e_{1}\right|^{\gamma}\left|\widetilde{e}_{2}\right|^{2-p / q}+\widetilde{e}_{2}^{2-p / q} \widetilde{e}_{1}^{y}\right)+\widetilde{\omega}(x, t) \\
& \left.\left.+k_{3}|\widetilde{s}|^{m}+k_{4}|\widetilde{s}|^{n / 2+1}\right)-k_{3} s^{m+1}-k_{4} s^{n / 2}|s|\right],
\end{aligned}
$$

where $s^{m+1}>0, s^{n / 2}|s|>0$.

The stability of ESO is obtained by selecting appropriate parameters $\beta_{1}, \beta_{2}$, and $\beta_{3}$. When the observer is stable, the derivative of $\varepsilon_{3}$ is 0 , and the error of $\omega(x, t)$ estimation can be written as

$$
\varepsilon_{3}=\beta_{2} \mathrm{fal}\left(\mathrm{fal}^{-1}\left(-\frac{h(t)}{\beta_{3}}\right), \alpha_{1}, \delta\right) .
$$

Note (11), if $\left|\varepsilon_{1}\right|>\delta$, the error of estimation is

$$
\left|\varepsilon_{3}\right|=\beta_{2}\left(\left|\frac{h(t)}{\beta_{3}}\right|\right)^{\alpha_{1} / \alpha_{2}} .
$$

And if $\left|\varepsilon_{1}\right| \leq \delta$, the error of estimation can be expressed as follows:

$$
\left|\varepsilon_{3}\right|=\frac{\beta_{2}}{\beta_{3}}\left(|h(t)| \delta^{\alpha_{1}-\alpha_{2}}\right) .
$$

From (32) and (33), it is clear that the estimation error $\varepsilon_{3}$ is determined by the parameters $\beta_{2}, \beta_{3}, \alpha_{1}, \alpha_{2}$, and $\delta$. The fundamental selection of the parameters can be chosen as $\beta_{2}>0, \beta_{3}>0,0<\alpha_{2}<\alpha_{1}<1$, and $\delta>0$. Furthermore, within the appropriate range, the larger $\beta_{3}$ is, the smaller $\left|h(t) / \beta_{3}\right|$ will be, although $h(t)$ is unknown to us. Of course, $\beta_{2}$ should be small enough to make the estimation error $\varepsilon_{3}$ as small as possible. Thus via turning these parameters properly, 


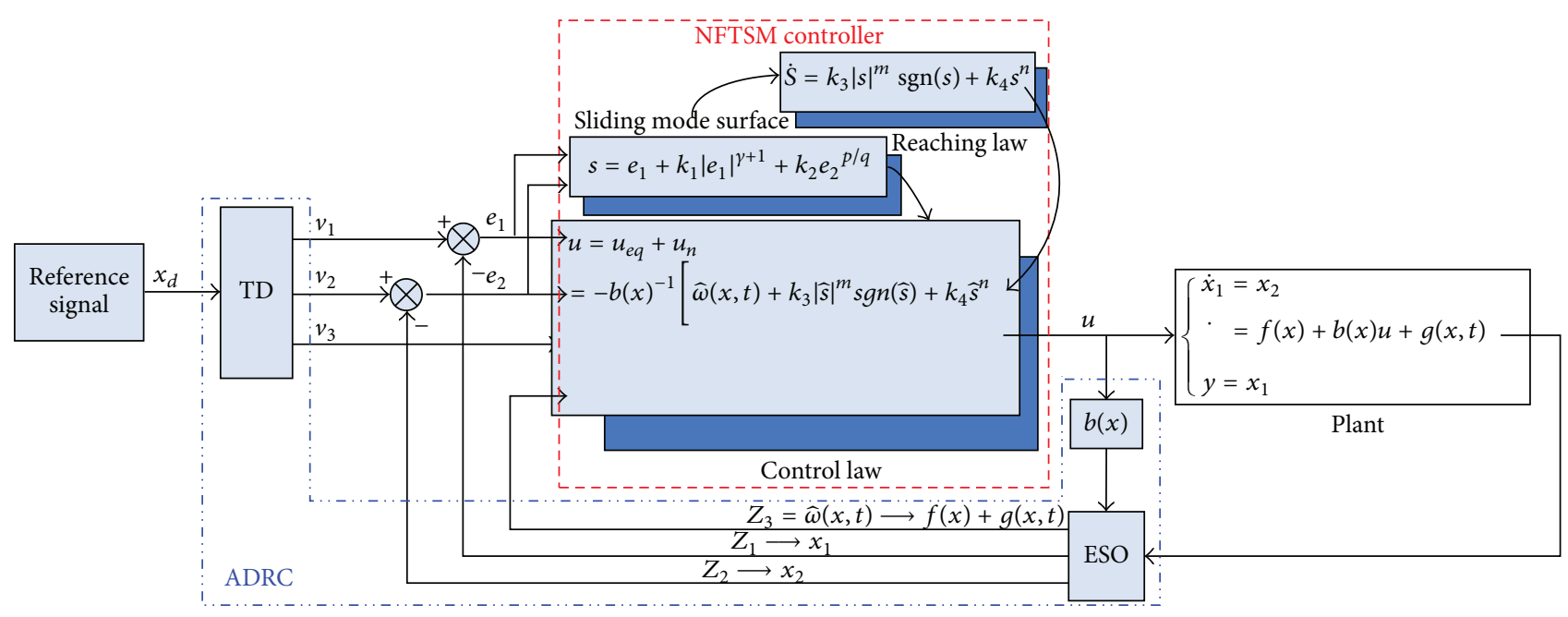

FIGURE 2: Complete structure of NFTSM control with ESO for uncertain nonlinear systems.

the estimation errors $|\widetilde{\omega}(x, t)|=\left|\varepsilon_{3}\right|$ will be small enough. $\widetilde{e}_{1}$ and $\widetilde{e}_{2}$ are the residual errors of states observer; $\widetilde{s}$ is the function of $\widetilde{e}_{1}$ and $\widetilde{e}_{2}$.

Suppose that

$$
\begin{aligned}
h= & \frac{1}{k_{2}} \frac{q}{p}\left|\widetilde{e}_{2}\right|^{2-p / q}+\frac{k_{1}(\gamma+1)}{k_{2}} \frac{q}{p} \\
& \times\left(\left|e_{1}\right|^{\gamma}\left|\widetilde{e}_{2}\right|^{2-p / q}+\widehat{e}_{2}^{2-p / q} \widetilde{e}_{1}^{\gamma}\right)+k_{3} \mid \widetilde{s}^{m} \\
& +k_{4}|\tilde{s}|^{n / 2+1},
\end{aligned}
$$

where $h$ is the function of the residual errors from ESO. For (30), we have

$$
\dot{V} \leq l\left(e_{2}\right)\left[s(h+\widetilde{\omega}(x, t))-k_{3} s^{m+1}-k_{4} s^{n / 2}|s|\right] .
$$

So, $\dot{V}<0$ can be satisfied when $k_{3}$ and $k_{4}$ are big enough. Therefore, $x_{1} \rightarrow x_{d}, x_{2} \rightarrow \dot{x}_{d}$, as $t \rightarrow t_{s}$.

3.4. Complete Structure of the Proposed Controller. The structure of NFTSM control with ESO is depicted in Figure 2.

From Figure 2, we can clearly see that the whole control is divided into two parts: NFTSM controller and ADRC. In this study, we innovatively combined these two parts.

\section{Numerical Simulation}

The numerical simulation results are presented to illustrate the feasibility and validity of the proposed control method in this section. Consider the following second-order SISO nonlinear system [13]:

$$
\begin{aligned}
\dot{x}_{1} & =x_{2}, \\
\dot{x}_{2} & =0.1 \sin (20 t)+u, \\
y & =x_{1},
\end{aligned}
$$

where $f(x)=0 ; g(x, t)=0.1 \sin (20 t) ; b(x)=1$.
TABLE 1: Control parameters of traditional NTSM controller and NTSM controller with exponent reaching law.

\begin{tabular}{lc}
\hline Parameters & Value \\
\hline$q$ & 3 \\
$p$ & 5 \\
$\beta$ & 1.0 \\
$k$ & 10 \\
$l_{g}$ & 0.015 \\
$\rho_{1}$ & 0.001 \\
$\rho_{2}$ & 0.2 \\
\hline
\end{tabular}

TABLE 2: Control parameters of the proposed NFTSM controller with ESO and TD.

\begin{tabular}{lc}
\hline Parameters & Value \\
\hline$q$ & 3 \\
$p$ & 5 \\
$\mathbf{k}=\left[k_{1}, k_{2}, k_{3}, k_{4}\right]$ & {$[0.7,0.8,150,150]$} \\
$m$ & 3 \\
$n$ & 3 \\
$\gamma$ & 6 \\
$\boldsymbol{\alpha}=\left[\alpha_{1}, \alpha_{2}\right]$ & {$[0.5,0.25]$} \\
$\boldsymbol{\beta}=\left[\beta_{1}, \beta_{2}, \beta_{3}\right]$ & {$[70,3200,10000]$} \\
$b_{0}=b$ & 1 \\
$\delta$ & 0.2 \\
$r$ & 10 \\
$h_{0}$ & 0.001 \\
\hline
\end{tabular}

For (36), the proposed NFTSM control method is compared with the NTSM control method [35] based on the exponent reaching law and the traditional NTSM control method [6], respectively.

(1) NTSM control method with exponent reaching law is as follows. 


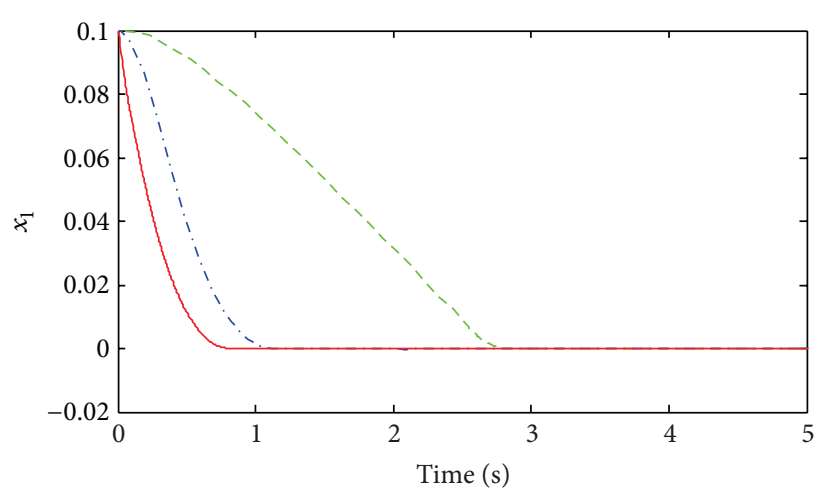

-. - The NTSM control with exponent reaching law

- - The traditional NTSM control

— The proposed NFTSM control with ESO

(a) $x_{1}$

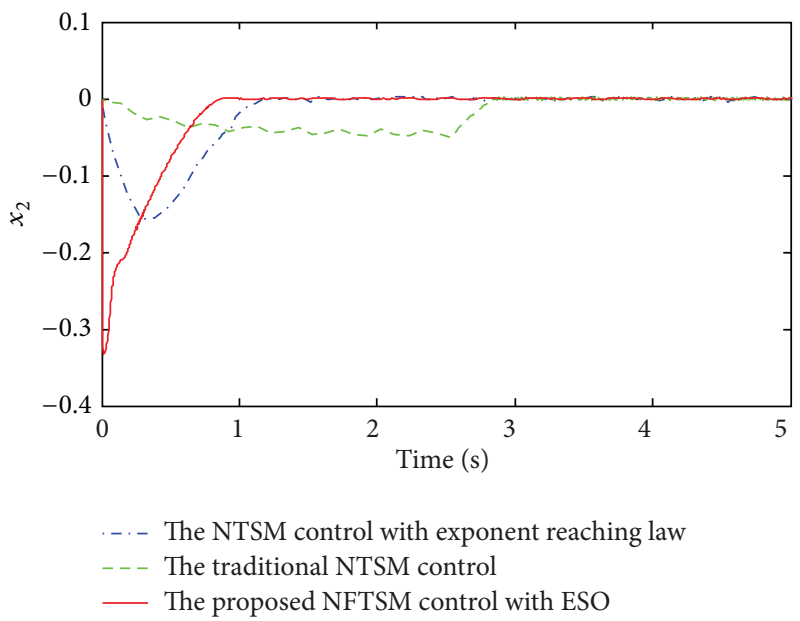

(b) $x_{2}$

FIGURE 3: States response comparison under three NTSM control methods.

The sliding mode surface, the reaching law, and the controller are given as

$$
\begin{gathered}
s=e_{1}+\frac{1}{\beta} e_{2}^{p / q}, \\
\dot{s}=-k s-\rho_{1} \operatorname{sgn}(s), \quad k>0, \rho_{1}>0, \\
u=-b(x)^{-1}\left[f(x)+\beta \frac{q}{p} e_{2}^{2-p / q}+k s\right. \\
\left.+\left(l_{g}+\rho_{1}\right) \operatorname{sgn}(s)-\ddot{x}_{d}\right] .
\end{gathered}
$$

(2) Traditional NTSM control method is as follows.

The sliding mode surface, the reaching law, and the controller are given as

$$
\begin{gathered}
s=e_{1}+\frac{1}{\beta} e_{2}^{p / q}, \\
\dot{s}=-\rho_{2} \operatorname{sgn}(s), \quad \rho_{2}>0, \\
u=-b(x)^{-1}\left[f(x)+\beta \frac{q}{p} e_{2}^{2-p / q}+\left(l_{g}+\rho_{2}\right) \operatorname{sgn}(s)-\ddot{x}_{d}\right] .
\end{gathered}
$$

4.1. Dynamic Performance Comparisons. To compare the control methods better, we select $\left[x_{1}(0), x_{2}(0)\right]=[0.1,0]$ as the initial values of system states. And the parameters of the three controllers are shown in Tables 1 and 2.

Figure 3 shows the states response comparison under NTSM with exponent reaching law, traditional NTSM, and NFTSM methods, respectively. Figure 4 shows TSM surface function $s$ comparison under three NTSM control methods.

From Figures 3 and 4, it can be clearly seen the states $x_{1}$, $x_{2}$, and $s$ under the proposed scheme are stabilized with the faster convergence speed and the shorter dynamical response time. A brief fluctuation occurs for $x_{2}$, whose maximum value

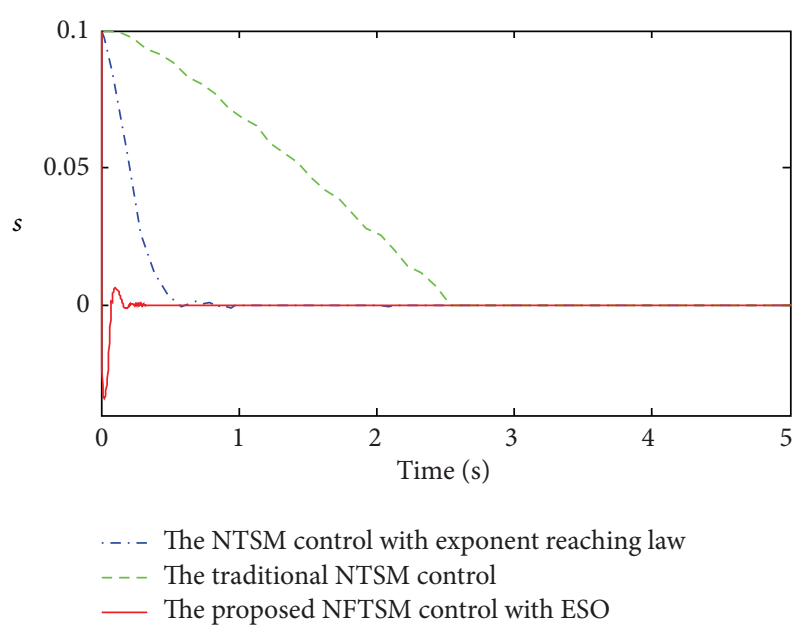

FIGURE 4: TSM surface function $s$ comparison under three NTSM control methods.

is about 0.32 , and the overshoot value is decreased to 0 rapidly. In addition, the steady state precision under the proposed scheme is satisfied despite the uncertainties and disturbances, which is higher than that of other methods. Figure 5 shows the control signal in the period of the system steady state (3s 5s).

From Figure 5, ESO can be used to estimate the uncertainty and disturbance and thus reduce the undesired chattering effectively.

To validate ESO performance, we present the states, observer values as well as the observer error of disturbance $g(x)$ (see Figures 6 and 7).

As depicted in Figure 6, ESO has the estimation ability of the system output $y=x_{1}$, and the steady observation error is within $4.5 \times 10^{-4}$. Based on the above analysis, the sliding mode surface $s$ is structured by the states errors $e_{1}$ and $e_{2}$. 


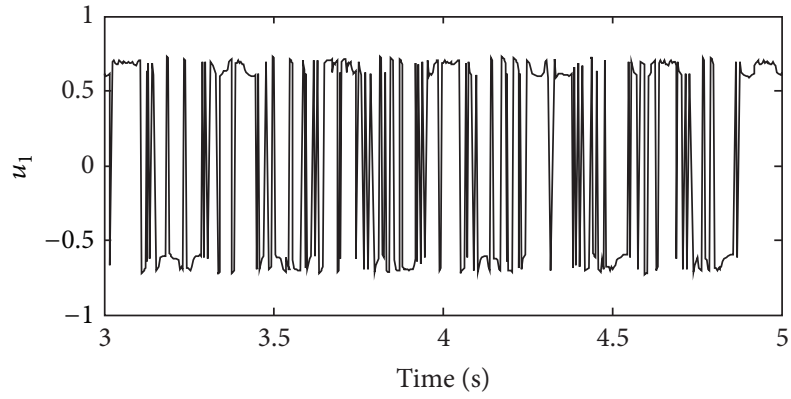

(a) NTSM controller $u_{1}$ with exponent reaching law

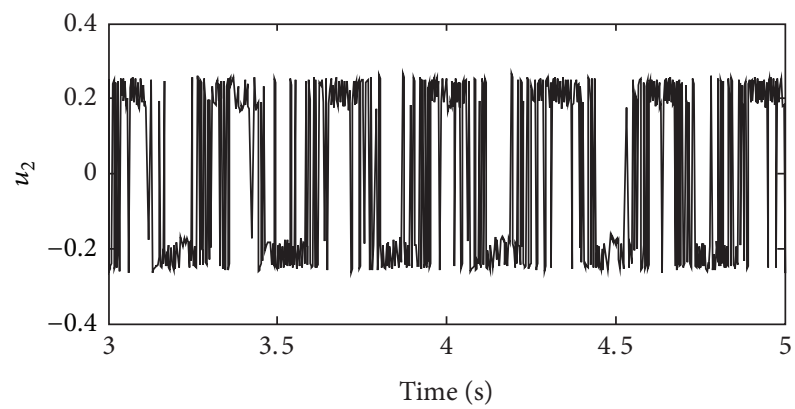

(b) Traditional NTSM controller $u_{2}$

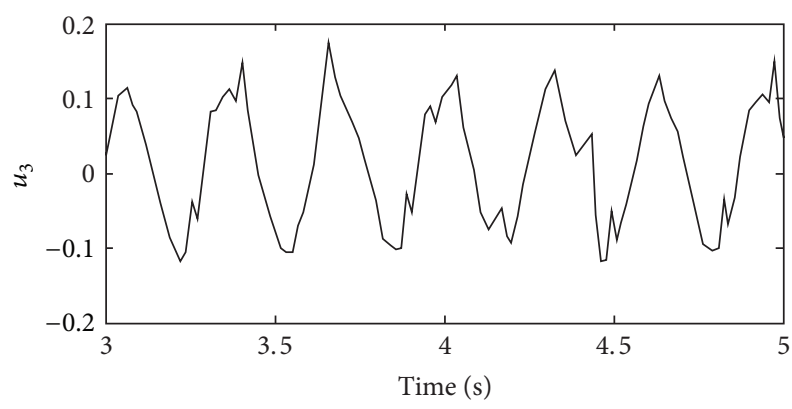

(c) Proposed NFTSM controller $u_{3}$ with ESO

FIGURE 5: Sliding mode surface function $s$ comparison under three NTSM control methods.

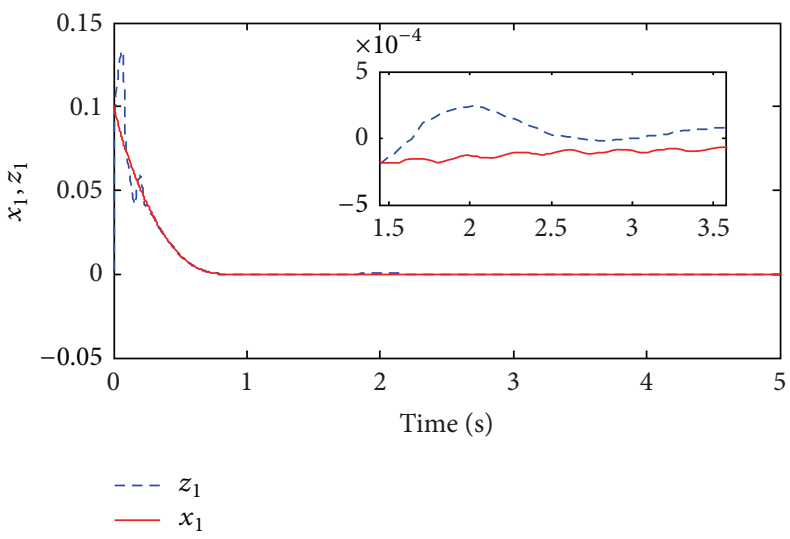

(a) $x_{1}$ and its observer value

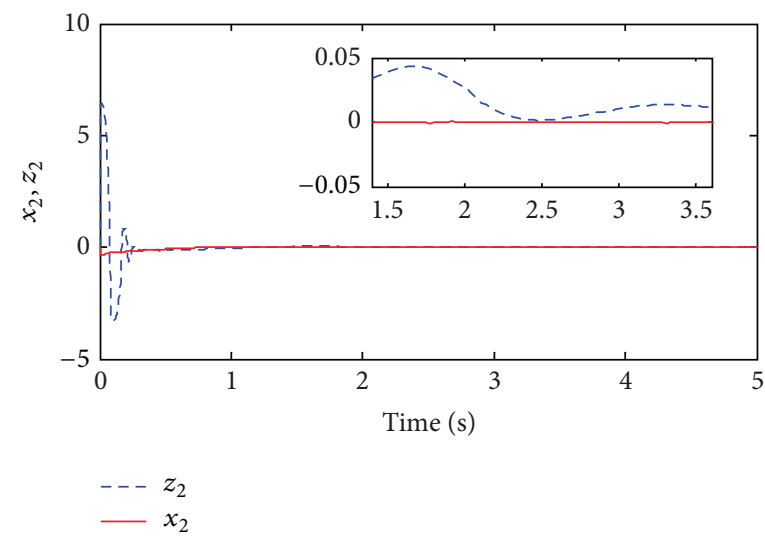

(b) $x_{2}$ and its observer value

Figure 6: System states and observer values via ESO.

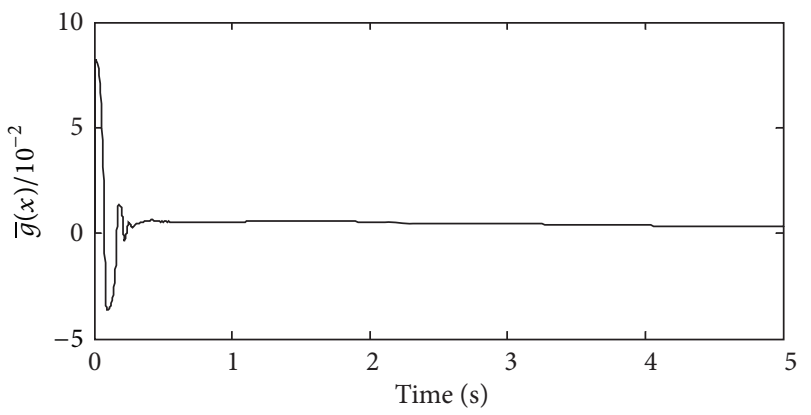

FIgURE 7: Observer error of the disturbance $g(x)$.
The chattering can be effectively reduced due to the small observer errors.

Furthermore, the proposed method will be compared with the ADRC method to show its superiority and reliability. As we know, ADRC consists of a tracking differentiator (TD), a nonlinear state-error feedback (NLSEF), and an extended state observer (ESO). The schematic diagram of ADRC is shown in Figure 8.

TD and ESO have been analyzed in the Section 2. In this section, nonlinear combination is introduced. State error feedback control law generates control voltage $u$ for system based on the error from the output of ESO and TD. The 


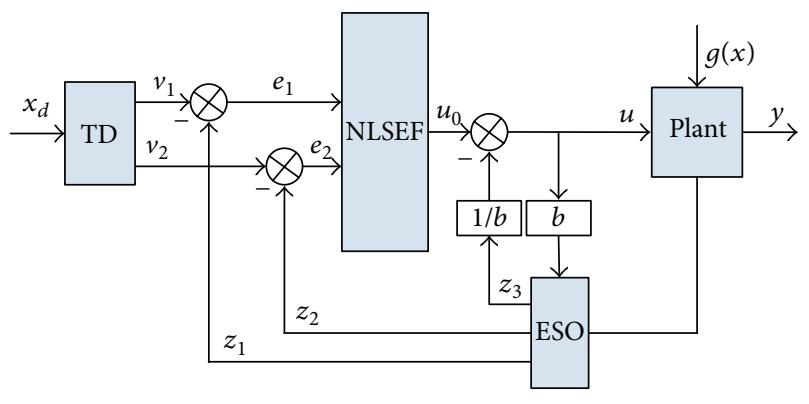

FIgURE 8: Schematic diagram of ADRC.

errors are combined with nonlinear manners; large errors correspond to lower gains and small errors correspond to higher gains. A typical nonlinear combination of error signal and its differential can be constructed as follows:

$$
u_{0}=l_{1} \mathrm{fal}\left(e_{1}, \alpha_{3}, \delta\right)+l_{2} \mathrm{fal}\left(e_{2}, \alpha_{4}, \delta\right),
$$

where $l_{1}$ and $l_{2}$ are proportional and differential coefficients, respectively.

The nonlinear function is used to make the observer more efficient. $e_{1}$ and $e_{2}$ are defined as follows:

$$
\begin{aligned}
& e_{1}=v_{1}-z_{1}, \\
& e_{2}=v_{2}-z_{2} .
\end{aligned}
$$

To enhance the performance, the nonlinear coefficients $\alpha_{1}$ and $\alpha_{2}$ are selected as $0<\alpha_{3}<1<\alpha_{4}$.

The controller of ADRC is designed as follows:

$$
u=u_{0}-\frac{z_{3}}{b_{0}}
$$

The control parameters of NLSEF are given in Table 3, and the parameters of ESO and TD are the same as the ones in Table 2.

Figure 9 shows the responses of system states $x_{1}$ and $x_{1}$ for system (1) under ADRC method. Control performances between proposed method and ADRC method is listed in Table 4.

Compared with the ADRC method, the proposed method can be used to reduce the steady states errors, eliminate its overshoot, and shorten its response time. The effects of the NFTSM with ESO and TD are improved based on the single NTSM control or the single ADRC.

4.2. Signal Tracking Analysis with the Noise. Consider the reference tracking signal with the noise signal:

$$
x_{d}=0.1 \sin (2 t)+\gamma n(t)
$$

where the noise $n(t)$ is a uniform distribution random variable varying in $[-1,1]$ at any given time $t$, which is independent with any other time; $\gamma$ is the noise intensity.

To show the superiority of TD, the control performances of the system (1) are compared by adopting the above three NTSM control schemes. We choose $\gamma$ with $0,0.0001$ and 0.001 respectively. The parameters of the three controllers are same as those of the above simulation tests in Tables 1 and 2. The dynamic control performances of the system and the noise suppression capability of TD are illustrated in Figures 10-12.

Figure 10 shows the system dynamic performances when the input reference signal is without the noise signal. Using three NTSM control, the system states $x_{1}, x_{2}$ can follow the ideal reference signal $x_{d}, \dot{x}_{d}$ after a certain time. But the system responses are the most quickly using the proposed NFTSM method with ESO and TD. Furthermore, the overshoot value of $u$ and the controller chattering are less than those of two other methods obviously.

Figures 11 and 12 show system dynamic control performances when $\gamma$ are 0.0001 and 0.001 . For the traditional NTSM control method, the $x_{1}$ tracking performance get badly quickly. Due to the adverse input noise and reasonless differentiator, $\dot{x}_{d}$ cannot be obtained effectively to worsen the $x_{2}$ tracking performance and intensify the controller chattering. TD of the proposed method shows the sufficient superiority. Although the controller chattering occurs, the maximal value can be limited in a small range about $[-5,5]$. Hence, the tracking performances of the system states and the noise restriction ability are satisfied in spite of the varying noise intensity $\gamma$ using the TD.

In summary, thanks to $\mathrm{TD}$, the useful input reference signal can be extracted and utilized to solve the required $n$ order differential value of $x_{d}$. This is an outstanding feather of ADRC design, and the reason why the stable tracking can be achieved with good transient quality and TD can be introduced to the proposed NFTSM control method to improve the control performances.

The simulation results can also show that the sin curve can be followed beautifully except for the constant value for the proposed control method.

4.3. Theodolite Rotating Control Example. Rotating servo system of electronic theodolite has been designed to achieve automatic searching and collimation of surveying object and accomplish automatic measuring with direct and inverted position of telescope. It consists of the telescope and sighting, the shafts, DC motor, and transmission device shown in Figure 13.

A DC torque motor is used for the vertical shaft driving device of theodolite rotating servo system. Considering the disturbances, the motor model can be composed by mechanical equation and electrical equation as follows:

$$
\begin{gathered}
J \ddot{\theta}=T_{e}-k_{v} \omega_{s}-T_{l}, \\
u=r_{a} i+L_{a} \frac{d i}{d t}+k_{e} \omega_{s}, \\
T_{e}=k_{T} i,
\end{gathered}
$$

where $r_{a}, L_{a}$, and $i$ represent armature resistance, inductance, and current, respectively; $k_{T}$ is the motor torque coefficient, $k_{v}$ is viscous friction coefficient, and $k_{e}$ is the counter voltage factor; $J$ denotes the total moment of inertia of motor $J_{m}$ and load $J_{l} ; T_{l}$ is the comprehensive disturbance including motor friction torque, system parameter uncertainty, and 


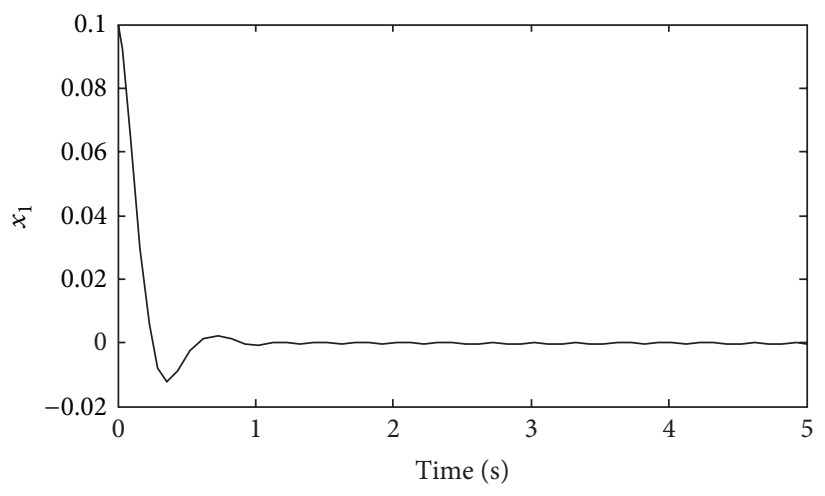

(a) $x_{1}$

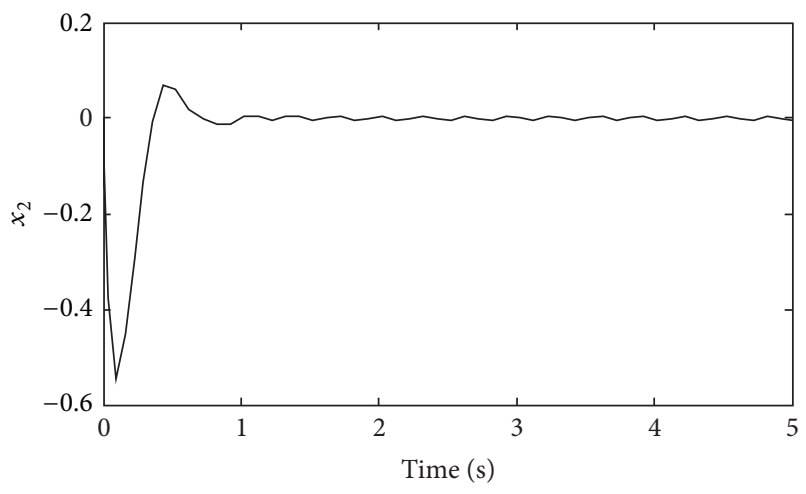

(b) $x_{2}$

FIgURE 9: System states under ADRC method.

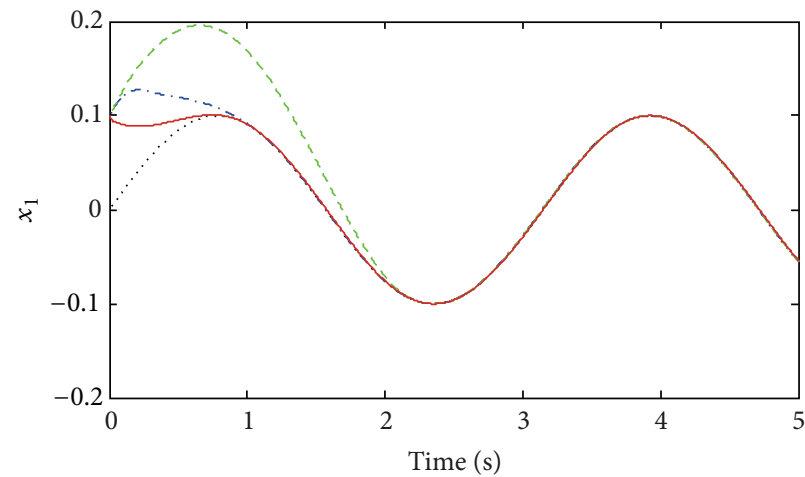

..... The ideal reference signal

. . . - The NTSM control with exponent reaching law

- - - The traditional NTSM control

_ The proposed NFTSM control

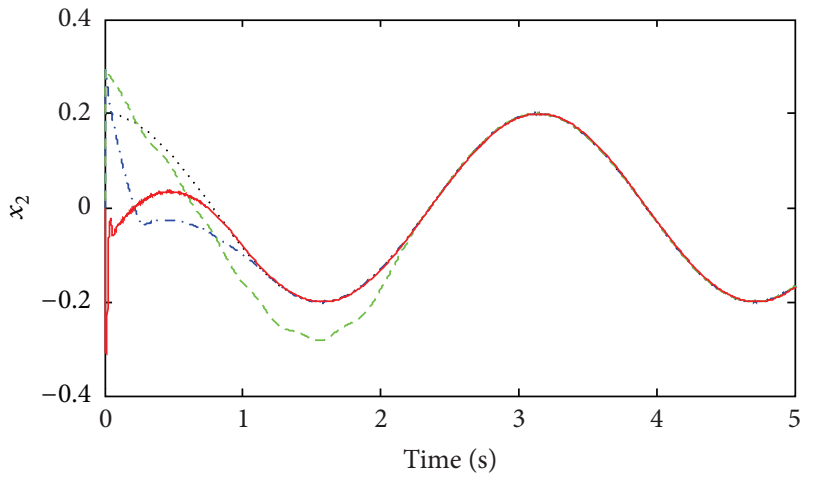

..... The ideal reference signal

... - The NTSM control with exponent reaching law

- . - The traditional NTSM control

_ The proposed NFTSM control

(a) $x_{1}$

(b) $x_{2}$

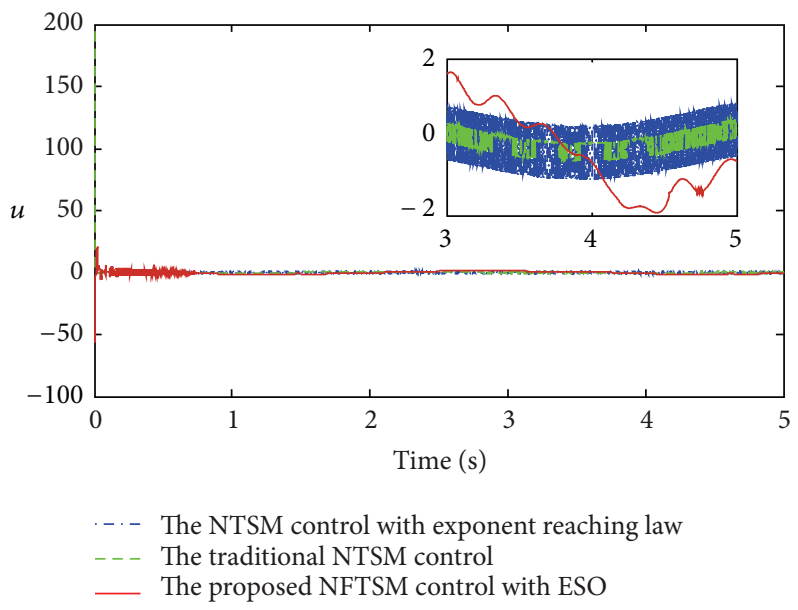

(c) Control input value $u$

FIGURE 10: System dynamic control performances when $\gamma$ is 0. 


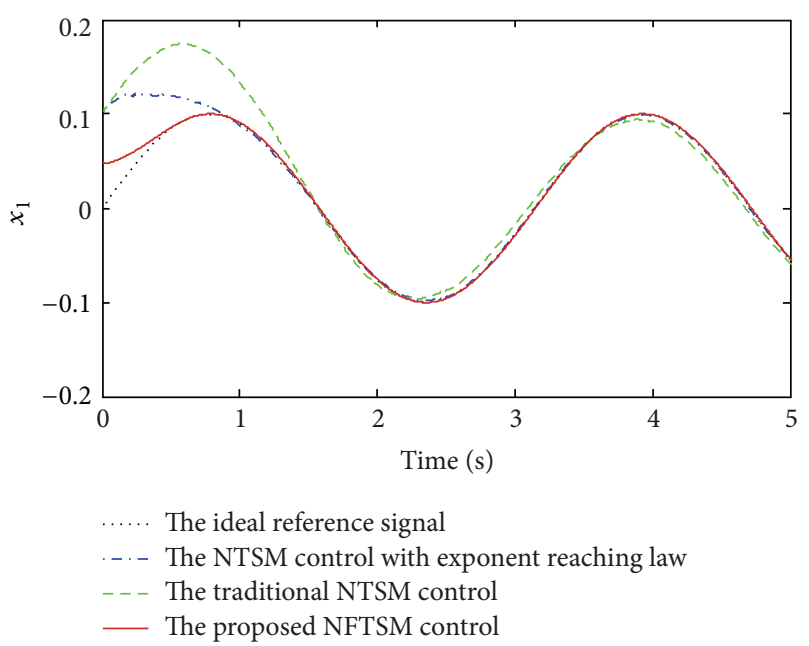

(a) $x_{1}$

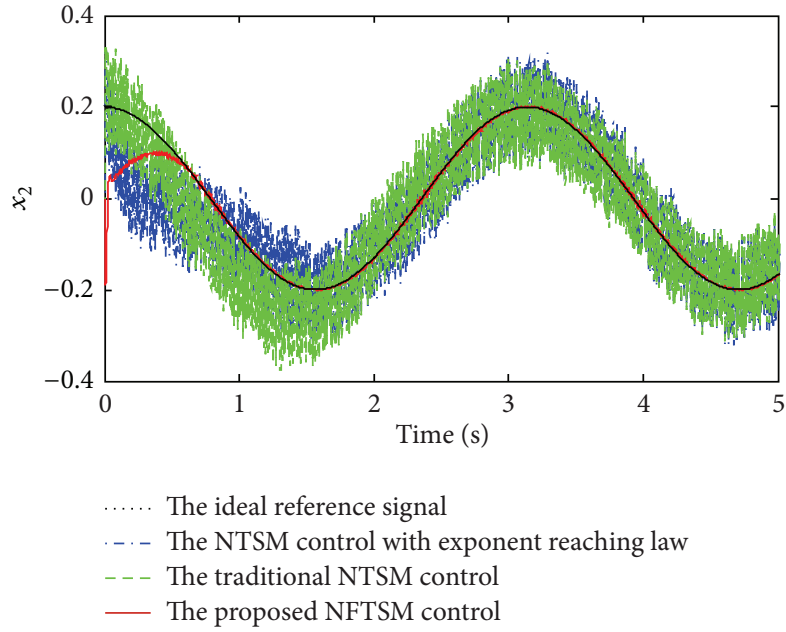

(b) $x_{2}$

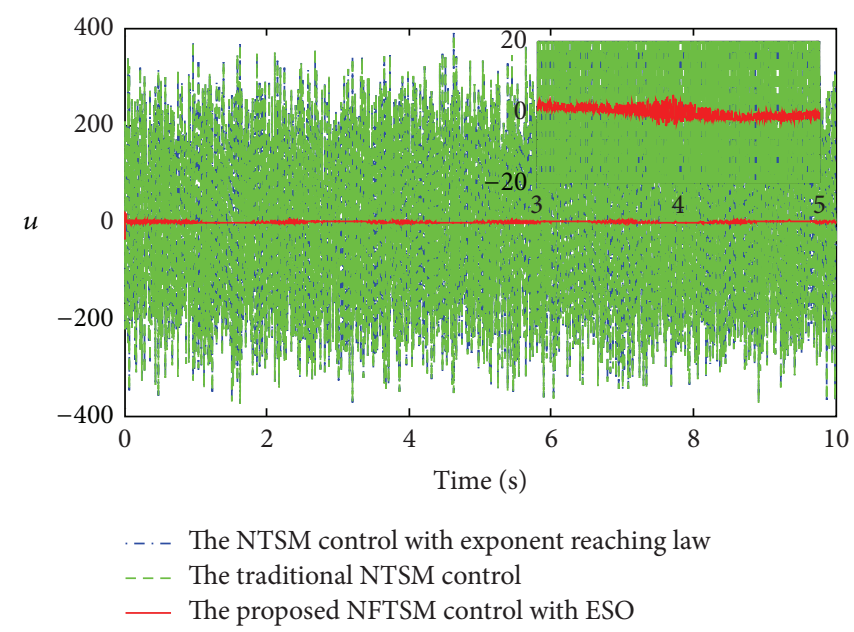

(c) Control input value $u$

FIGURE 11: System dynamic control performances when $\gamma$ is 0.0001 .

other disturbance; $T_{e}$ is the motor output torque; $u$ is the controller output; $\theta$ is the actual angular position of motor; $\omega_{s}$ is the actual angular velocity of motor.

With the small $L_{a}$, theodolite rotating servo system can be simplified as follows:

$$
\begin{gathered}
\dot{\theta}=\omega_{s}, \\
\dot{\omega}_{s}=a \omega_{s}+b u(t)-\frac{T_{f}\left(\omega_{s}\right)}{J}+d(t),
\end{gathered}
$$

where $a=-\left(k_{e} k_{T} /\left(r_{a} J\right)+k_{v} / J\right), b=k_{T} /\left(r_{a} J\right) . T_{f}\left(\omega_{s}\right)$ denotes the friction torque, and $d(t)$ denotes the external kinematic disturbance.

In this paper, we choose a familiar friction model considering the system static friction, coulomb friction, and the Stribeck effect [36]. The friction model can be described as follows:

$$
T_{f}=f_{c}+\left(f_{s}-f_{c}\right) \exp \left(-\left|\frac{\omega_{s}}{\omega_{0}}\right|^{2}\right)
$$

where $\omega_{0}$ is Stribeck velocity; $f_{c}$ and $f_{s}$ represent the levels of Coulomb friction and stiction, respectively.

Define the angular position and angular velocity as the system state variables; that is, $\left[x_{1}, x_{2}\right]^{T}=\left[\theta, \omega_{s}\right]^{T}$, so theodolite rotating control system can be expressed as follows:

$$
\begin{aligned}
\dot{x}_{1} & =x_{2}, \\
\dot{x}_{2} & =a x_{2}+g\left(x_{2}\right)+b u(t)+d(t), \\
y & =x_{1},
\end{aligned}
$$

where $x_{1}=y=\theta$ is output variable and $d(t)$ is the external disturbance. $g\left(x_{2}\right)=T_{f}\left(\omega_{s}\right) / J$. Define the error $e_{r}=x_{d}-x_{1}$, where $x_{d}$ is target angular position.

To obtain better rotating speed, accuracy, and stability, the trajectory planning need to be carried out in the process of theodolite rotating to determine a reasonable angular displacement function. This paper presents thirdorder trajectory planning algorithm with a uniform presence 


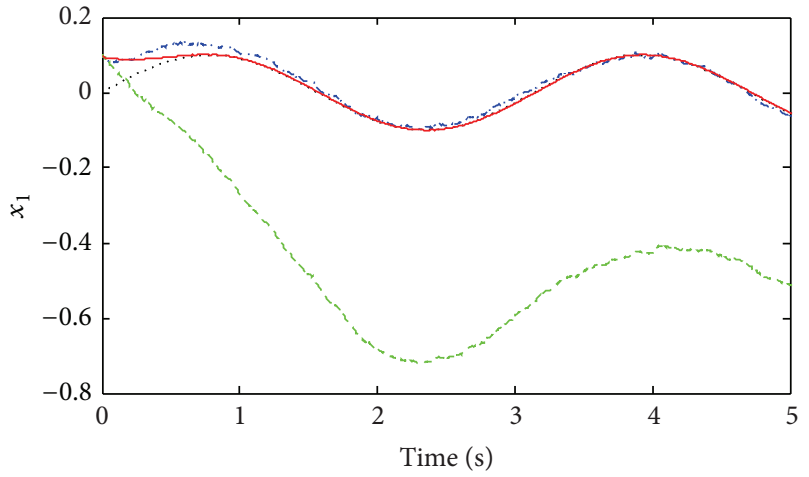

.... The ideal reference signal

...- The NTSM control with exponent reaching law

- - - The traditional NTSM control

— The proposed NFTSM control

(a) $x_{1}$

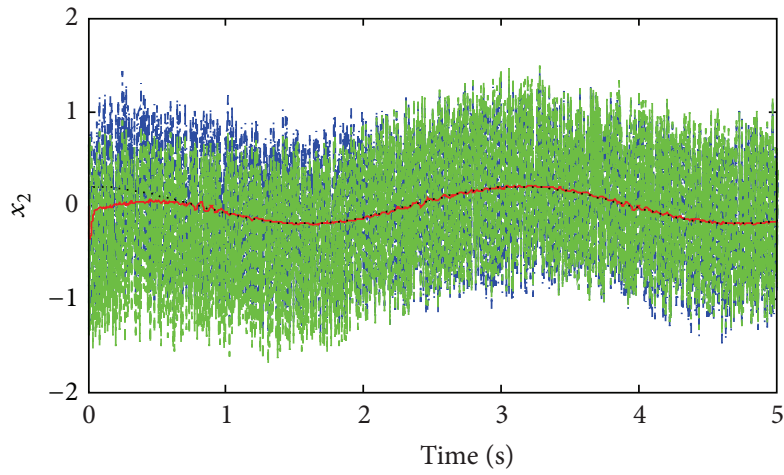

The ideal reference signal

- - The NTSM control with exponent reaching law

- - - The traditional NTSM control

— The proposed NFTSM control

(b) $x_{2}$

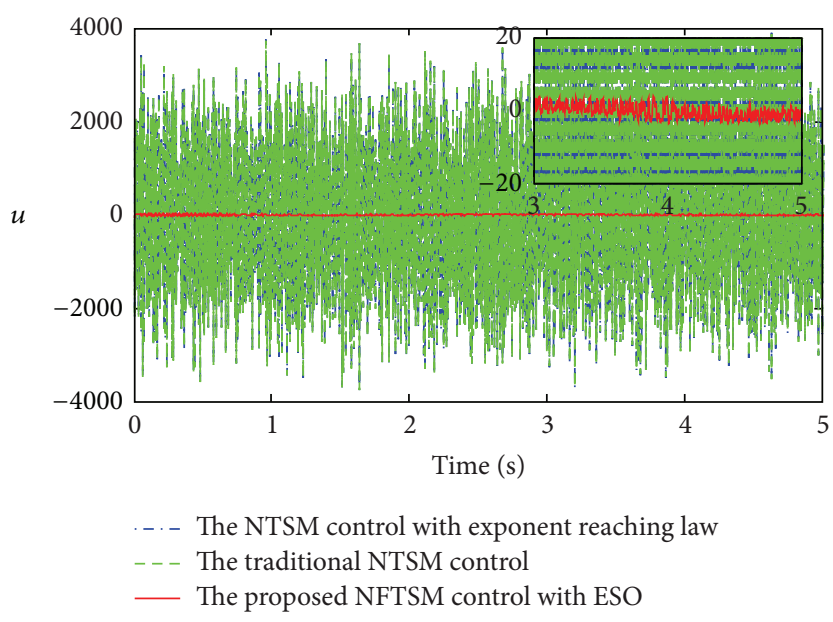

(c) Control input value $u$

FIGURE 12: System dynamic control performances when $\gamma$ is 0.001 .

segment for point-point precision rotating of theodolite [37]. By trajectory planning, the ideal goal curve of theodolite azimuth angle can be expressed as follows:

$$
\theta_{d}= \begin{cases}\frac{64}{3} t^{3} & 0 \leq t \leq \frac{1}{8} \\ -\frac{64}{3}\left(t-\frac{1}{8}\right)^{3}+8\left(t-\frac{1}{8}\right)^{2}+\left(t-\frac{1}{12}\right) & \frac{1}{8} \leq t \leq \frac{1}{4} \\ 2\left(t-\frac{1}{4}\right)+\frac{1}{4} & \frac{1}{4} \leq t \leq \frac{3}{4} \\ -\frac{64}{3}\left(t-\frac{3}{4}\right)^{3}+2\left(t-\frac{3}{4}\right)+\frac{5}{4} & \frac{3}{4} \leq t \leq \frac{7}{8} \\ \frac{64}{3}\left(t-\frac{7}{8}\right)^{3}-8\left(t-\frac{7}{8}\right)^{2}+\left(t+\frac{1}{6}\right) & \frac{7}{8} \leq t \leq 1 .\end{cases}
$$

Furthermore, the reference curve $x_{d}$ of rotor angular displacement can be obtained according to $\theta_{d}$ and transmission
TABLE 3: Control parameters of NLSEF.

\begin{tabular}{lc}
\hline Parameters & Value \\
\hline$l=\left[l_{1}, l_{2}\right]$ & {$[3,5]$} \\
$\boldsymbol{\alpha}=\left[\alpha_{3}, \alpha_{4}\right]$ & {$[0.75,1.25]$} \\
$\boldsymbol{b}_{0}=b$ & 1 \\
\hline
\end{tabular}

ratio. The system parameters are listed in Table 5. The friction parameters are obtained by offline system identification.

To evaluate the efficiency of proposed control method, the SMC based on the exponential reaching law and PID control methods are also employed in the simulations for the purpose of comparisons. The parameters of the proposed controller are as follows:

$k_{3}=150 ; k_{4}=220 ; \boldsymbol{\beta}=\left[\beta_{1}, \beta_{2}, \beta_{3}\right]=[35,150,6500] ;$ $b_{0}=0.362$. The other parameters of proposed controller are the same as those in Table 2. 
TABLE 4: Control performance comparisons between the proposed method and the ADRC method.

\begin{tabular}{lcc}
\hline Parameters & Proposed method & ADRC method \\
\hline Steady state error of $x_{1}$ & $1.1 \times 10^{-4}$ & $4.2 \times 10^{-4}$ \\
Steady state error of $x_{2}$ & $1.0 \times 10^{-3}$ & $5.9 \times 10^{-3}$ \\
Max. overshoot of $x_{1}$ & 0 & 0.012 \\
Max. overshoot of $x_{2}$ & 0.33 & 0.54 \\
Response time & 0.84 & 1.2 \\
\hline
\end{tabular}

TABLE 5: The main parameters of theodolite rotating system.

\begin{tabular}{lcc}
\hline System parameters & Symbol & Size \\
\hline Rated motor voltage/V & $U_{0}$ & 9 \\
Motor armature resistance/ $\Omega$ & $r_{a}$ & 2.76 \\
Motor torque coefficient/Nm/A & $k_{T}$ & 1.2 \\
Viscous friction coefficient & $k_{v}$ & 0.2 \\
Counter voltage factor & $k_{e}$ & 0.7 \\
System moment of inertia/Kgm ${ }^{2}$ & $J$ & 0.2 \\
Coulomb friction/Nm & $f_{c}$ & 2.0 \\
Coulomb stiction/Nm & $f_{s}$ & 2.6 \\
Stribeck velocity & $\omega_{0}$ & 0.06 \\
Number of pole pairs & $P$ & 4 \\
Motor EMF coefficient/V/(rad/s) & $k_{e}$ & 0.7 \\
Sampling time/sec & $T$ & 0.001 \\
\hline
\end{tabular}

In the comparing simulation, PID controller is designed as follows:

$$
u(t)=k_{p} e(t)+k_{i} \int e(t) d t+k_{d} \dot{e}(t)
$$

where proportion coefficient $k_{p}=200$, integral coefficient $k_{i}=5$, and differential coefficient $k_{d}=5 \times 10^{-4}$ by using cut-and-try method.

The conventional sliding mode control adopts SMC based on exponential reaching law, and the designed reaching law is given as follows:

$$
\dot{s}=-\delta \operatorname{sgn}(s)-k s, \quad \varepsilon>0, k>0,
$$

where $\delta=5.1 \times 10^{5}$, and $k=2.2$.

So the controller is shown in

$$
u_{2}(t)=a_{3}\left[k_{1} e_{2}+\ddot{x}_{1 d}-a x_{2}-g\left(x_{2}\right)+k s+\delta \operatorname{sgn}(s)\right],
$$

where $s=k_{1} e_{1}+e_{2} ; e_{1}=x_{1 d}-x_{1} ; e_{2}=\dot{x}_{1 d}-x_{2} ; k_{1}=350$.

Due to the periodicity in rotating system, the lumped uncertainties $d(t)$ can be supposed for $-0.5 \sin (\pi t)$, and the initial values of the system are $\left[x_{1}, x_{2}\right]=[0,0]$. Three controllers are designed to control theodolite rotating system. respectively, and the results are shown in Figures 14-17.

The angle tracking accuracy and the positioning accuracy are two important indexes for theodolite rotating systems. Figure 14 shows that three controllers can track well the target angle curve by adjusting parameter. From Figure 15, the tracking precision with the proposed SMC method is

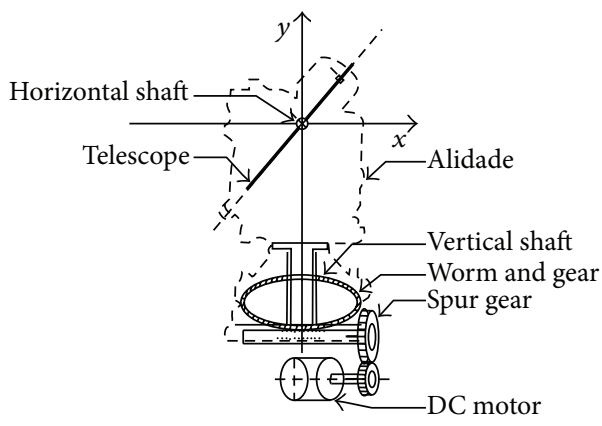

FIGURE 13: The rotating servo system of automatic electronic theodolite.

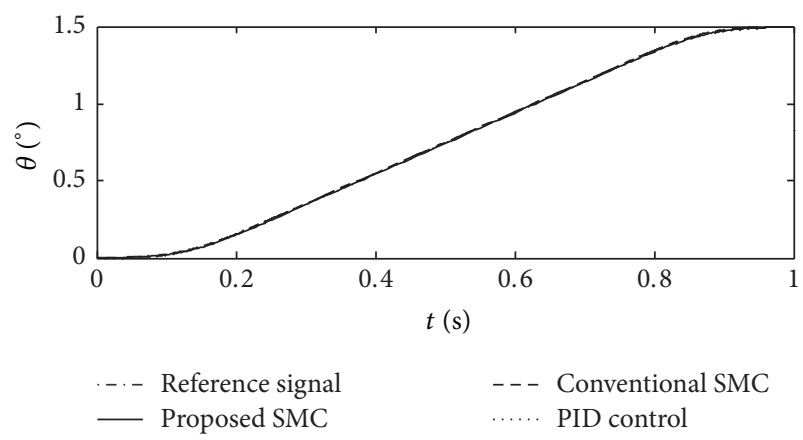

FIGURE 14: Comparison of rotating angle curves.

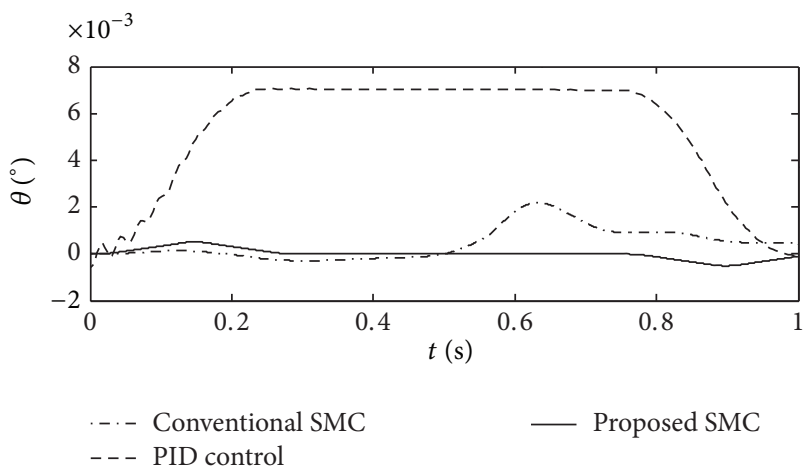

FIGURE 15: Tracking error curves.

better than the other two control methods. Since the integral term is introduced in the controller, the static error using the proposed control method can limit at $5.6 \times 10^{-60}$; the maximum tracking angle error is about $5.1 \times 10^{-5 \circ}$; the final positioning error after rotating is about $2.5 \times 10^{-40}$; with the system interference and the uncertainty, the designed sliding mode controller can quickly stabilize the target signal.

Speed stability is also an important performance index for theodolite rotating, which directly affects CCD image quality and causes mechanical vibration of theodolite. Rotating angle speed curves are shown in Figure 16.

From Figure 16, by comparing three controllers, the speed tracking curve is the best adopting the proposed method. The maximum tracking error of rotating speed is about $1.82^{\prime \prime} / \mathrm{s}$, and rotating speed fluctuation value is less than $2.5 \%$. Partial 


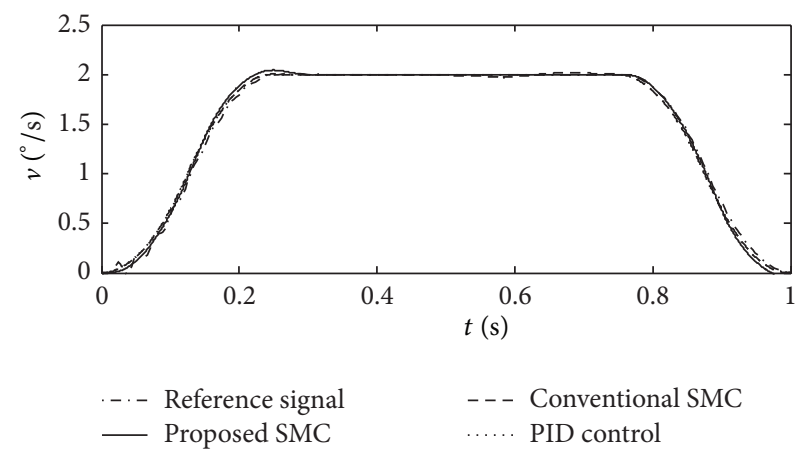

(a) Angle speed curves

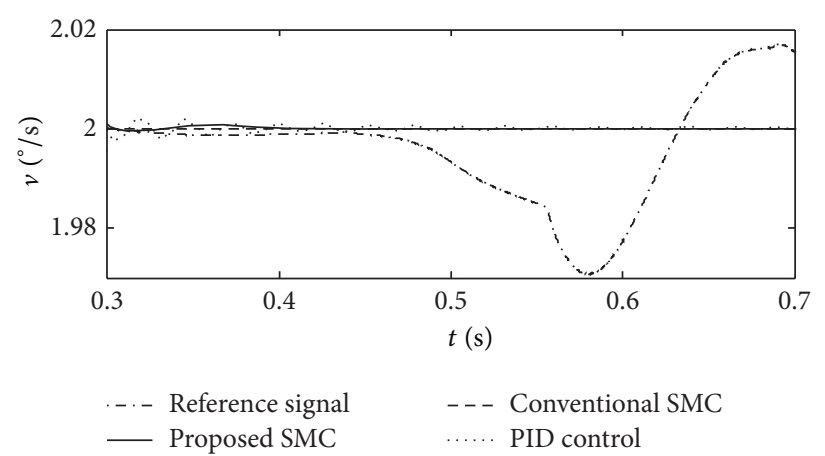

(b) Local zoom curves in speed steady state

FIGURE 16: Comparison of angle speed curves.

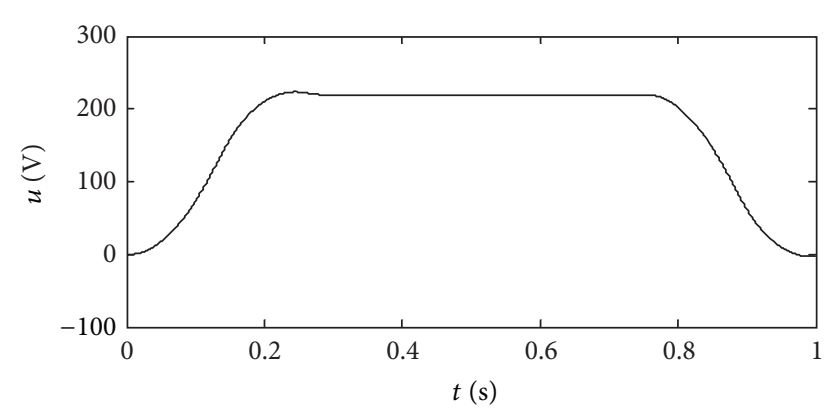

FIGURE 17: Output of proposed sliding mode controller.

enlarged steady speed shows that the system states cannot convergence effectively by the conventional sliding mode and the speed tracking error is larger. For PID control method, the target speed curve can be tracked, but there are significant speed fluctuations.

Figure 17 shows the controller output curve is smooth, and overcomes the chattering. In addition, the control input of any practical system is bounded. We design the rational control parameters to ensure the physical implementation of the controller and the tolerance range of the actuator.

\section{Conclusion}

In this paper, a new continuous terminal fast sliding mode controller with ESO and TD is proposed for nonlinear uncertain systems, based on an extended state observer which is used to estimate the disturbances and the states. In order to get better control performance, an improved nonsingular fast terminal sliding mode surface and a novel global fast reaching law are designed in the controller. Under the proposed terminal sliding mode controller, we know that the finite time convergence to the sliding mode is guaranteed. As compared with the traditional TSMC and ADRC, the proposed controller has exhibited three superiorities in the simulation including faster convergence speed, better dynamic performance and weakened chattering effect, and noise pollution of the reference input. Simulation results of theodolite rotating system have also demonstrated that the proposed method has good dynamic and static performances to satisfy the requirement of precise angle measurement.

\section{Conflict of Interests}

The authors declare that there is no conflict of interests regarding the publication of this paper.

\section{Acknowledgment}

The authors gratefully acknowledge the support for the realization of this work from the following foundation: National Nature Science Foundation of China under Grant 41174162.

\section{References}

[1] V. I. Utkin, "Sliding mode control design principles and applications to electric drives," IEEE Transactions on Industrial Electronics, vol. 40, no. 1, pp. 23-36, 1993.

[2] H. Sun, S. Li, and C. Sun, "Finite time integral sliding mode control of hypersonic vehicles," Nonlinear Dynamics, vol. 73, no. 1-2, pp. 229-244, 2013.

[3] K. Lu and Y. Xia, "Adaptive attitude tracking control for rigid spacecraft with finite-time convergence," Automatica, vol. 49, no. 12, pp. 3591-3599, 2013.

[4] M. Zak, "Terminal attractors in neural networks," Physics Letters, vol. 2, no. 4, pp. 259-274, 1989.

[5] M. Zhihong, A. P. Paplinski, and H. R. Wu, "A robust MIMO terminal sliding mode control scheme for rigid robotic manipulators," IEEE Transactions on Automatic Control, vol. 39, no. 12, pp. 2464-2469, 1994.

[6] M. Zhihong and X. H. Yu, "Terminal sliding mode control of MIMO linear systems," IEEE Transactions on Circuits and Systems. I. Fundamental Theory and Applications, vol. 44, no. 11, pp. 1065-1070, 1997.

[7] K.-B. Park and J.-J. Lee, "Comments on 'a robust MIMO terminal sliding mode control scheme for rigid robotic manipulators"' IEEE Transactions on Automatic Control, vol. 41, no. 5, pp. 761-762, 1996.

[8] Y. Feng, X. Yu, and Z. Man, "Non-singular terminal sliding mode control of rigid manipulators," Automatica, vol. 38, no. 12, pp. 2159-2167, 2002. 
[9] X. Yu and M. Zhihong, "Fast terminal sliding-mode control design for nonlinear dynamical systems," IEEE Transactions on Circuits and Systems. I. Fundamental Theory and Applications, vol. 49, no. 2, pp. 261-264, 2002.

[10] S. Li, K. Li, J. Wang et al., "Nonsingular and fast terminal sliding mode control method," Information and Control, vol. 389, no. 1, pp. 1-8, 2009.

[11] J.-J. Slotine and S. S. Sastry, "Tracking control of nonlinear systems using sliding surfaces, with application to robot manipulators," International Journal of Control, vol. 38, no. 2, pp. 465492, 1983.

[12] I.-C. Baik, K.-H. Kim, and M.-J. Youn, "Robust nonlinear speed control of PM synchronous motor using boundary layer integral sliding mode control technique," IEEE Transactions on Control Systems Technology, vol. 8, no. 1, pp. 47-54, 2000.

[13] A. Bagheri and J. J. Moghaddam, "Simulation and tracking control based on neural-network strategy and sliding-mode control for underwater remotely operated vehicle," Neurocomputing, vol. 72, no. 7-9, pp. 1934-1950, 2009.

[14] J. Yang, J. Su, S. Li, and X. Yu, "High-order mismatched disturbance compensation for motion control systems via a continuous dynamic sliding-mode approach," IEEE Transactions on Industrial Informatics, vol. 10, no. 1, pp. 604-614, 2014.

[15] K. Ohnishi, "A new servo method inmechatronics," Transactions of the Japanese Society of Electrical Engineers, vol. 107D, pp. 83-86, 1987.

[16] X. Wei and L. Guo, "Composite disturbance-observer-based control and terminal sliding mode control for non-linear systems with disturbances," International Journal of Control, vol. 82, no. 6, pp. 1082-1098, 2009.

[17] X. Wei, H. Zhang, and L. Guo, "Composite disturbanceobserver-based control and variable structure control for nonlinear systems with disturbances," Transactions of the Institute of Measurement and Control, vol. 31, no. 5, pp. 401-423, 2009.

[18] X. Wei, H.-F. Zhang, and L. Guo, "Composite disturbanceobserver-based control and terminal sliding mode control for uncertain structural systems," International Journal of Systems Science, vol. 40, no. 10, pp. 1009-1017, 2009.

[19] J. Yang, S. Li, and X. Yu, "Sliding-mode control for systems with mismatched uncertainties via a disturbance observer," IEEE Transactions on Industrial Electronics, vol. 60, no. 1, pp. 160-169, 2013.

[20] J. Yang, S. Li, J. Su, and X. Yu, "Continuous nonsingular terminal sliding mode control for systems with mismatched disturbances," Automatica, vol. 49, no. 7, pp. 2287-2291, 2013.

[21] J. Su, J. Yang, and S. Li, "Continuous finite-time anti-disturbance control for a class of uncertain nonlinear systems," Transactions of the Institute of Measurement and Control, vol. 36, no. 3, pp. 300-311, 2014.

[22] J. Han, "Active disturbance rejection controller and its applications," Control and Decision, vol. 13, no. 1, pp. 19-23, 1998.

[23] Z.-Q. Gao, "On the foundation of active disturbance rejection control," Control Theory \& Applications, vol. 30, no. 12, pp. 14981510, 2013.

[24] Y. Huang and W. C. Xue, "Active disturbance rejection control: methodology, applications and theoretical analysis," Journal of Systems Science and Mathematical Sciences, vol. 32, no. 10, pp. 1287-1307, 2012.

[25] Y.-Q. Xia, M.-Y. Fu, Z.-H. Deng, and X.-M. Ren, "Recent developments in sliding mode control and active disturbance rejection control," Control Theory \& Applications, vol. 30, no. 2, pp. 137-147, 2013.
[26] P.-P. Wang and H. Zhou, "Electronic laser theodolite automatic targeting method based on scan-line model," Opto-Electronic Engineering, vol. 39, no. 1, pp. 103-107, 2012.

[27] J. A. Ratches, "Review of current aided/automatic target acquisition technology for military target acquisition tasks," Optical Engineering, vol. 50, no. 7, Article ID 072001, 2011.

[28] C. Zhao, Y. Pan, C. Ren, B. Ma, and H. Luo, "The control of position transformation on north seeker based on adaptive fuzzy-PID," Piezoelectrics and Acoustooptics, vol. 34, no. 1, pp. 51-55, 2012.

[29] K. Wang, X. Guo, Z. Zhou et al., "Research of accurate theodolite rotating technology based on PIDNN," Computer Measurement and Control, vol. 20, no. 11, pp. 3042-3047, 2012.

[30] Z. Gao, "Scaling and bandwidth-parameterization based controller tuning," in Proceedings of the American Control Conference, pp. 4989-4996, Denver, Colo, USA, June 2003.

[31] J. Han, "A class of extended state observer for uncertain systems," Control Decision, vol. 10, no. 1, pp. 85-88, 1995.

[32] S.-B. Li, K.-Q. Li, J.-Q. Wang, and B. Yang, "Nonsingular fast terminal-sliding-mode control method and its application on vehicular following system," Control Theory and Applications, vol. 27, no. 5, pp. 543-550, 2010.

[33] S.-F. Xiong, W.-H. Wang, and S. Wang, "Nonsingular fast terminal sliding-mode guidance with intercept angle constraint," Control Theory and Applications, vol. 31, no. 3, pp. 269-278, 2014.

[34] Y. Moreno, M. Nekovee, and A. F. Pacheco, "Dynamics of rumor spreading in complex networks," Physical Review E: Statistical, Nonlinear, and Soft Matter Physics, vol. 69, Article ID 066130, 2004.

[35] W. W. Zhang and J. Wang, "Nonsingular terminal sliding mode control based on exponential reaching law," Control and Decision, vol. 27, no. 6, pp. 909-913, 2012.

[36] Y. Song, H.-B. Gao, S.-M. Zhang, B.-X. Du, and T.-J. Guo, "Adaptive compensation of torque ripple in DC torque motor," Optics and Precision Engineering, vol. 18, no. 10, pp. 2212-2220, 2010.

[37] H. Mu, Y. Zhou, S. Yan, and Y. Hua, "A third-order trajectory planning algorithm for ultra-precision point-to-point motion," Mechanical Science and Technology for Aerospace Engineering, vol. 27, no. 2, pp. 234-240, 2008. 


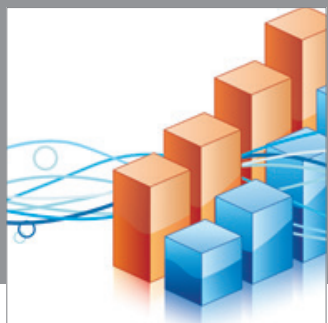

Advances in

Operations Research

mansans

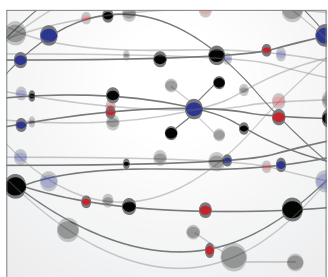

The Scientific World Journal
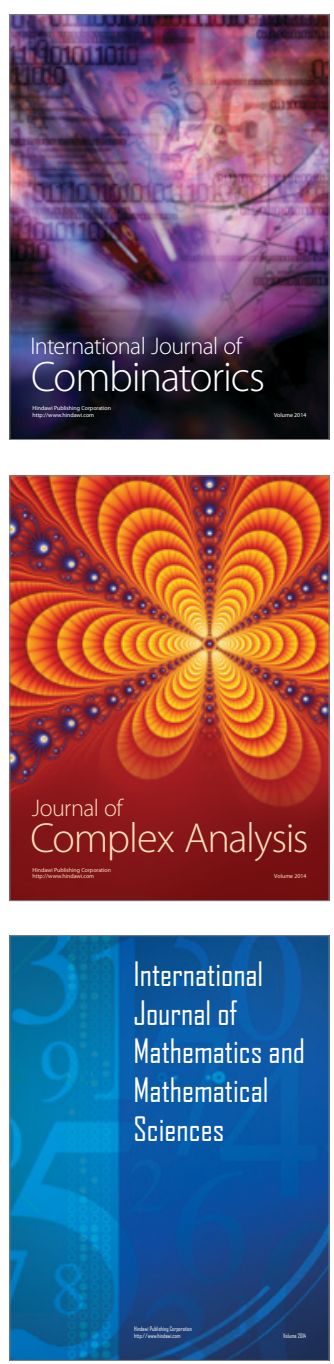
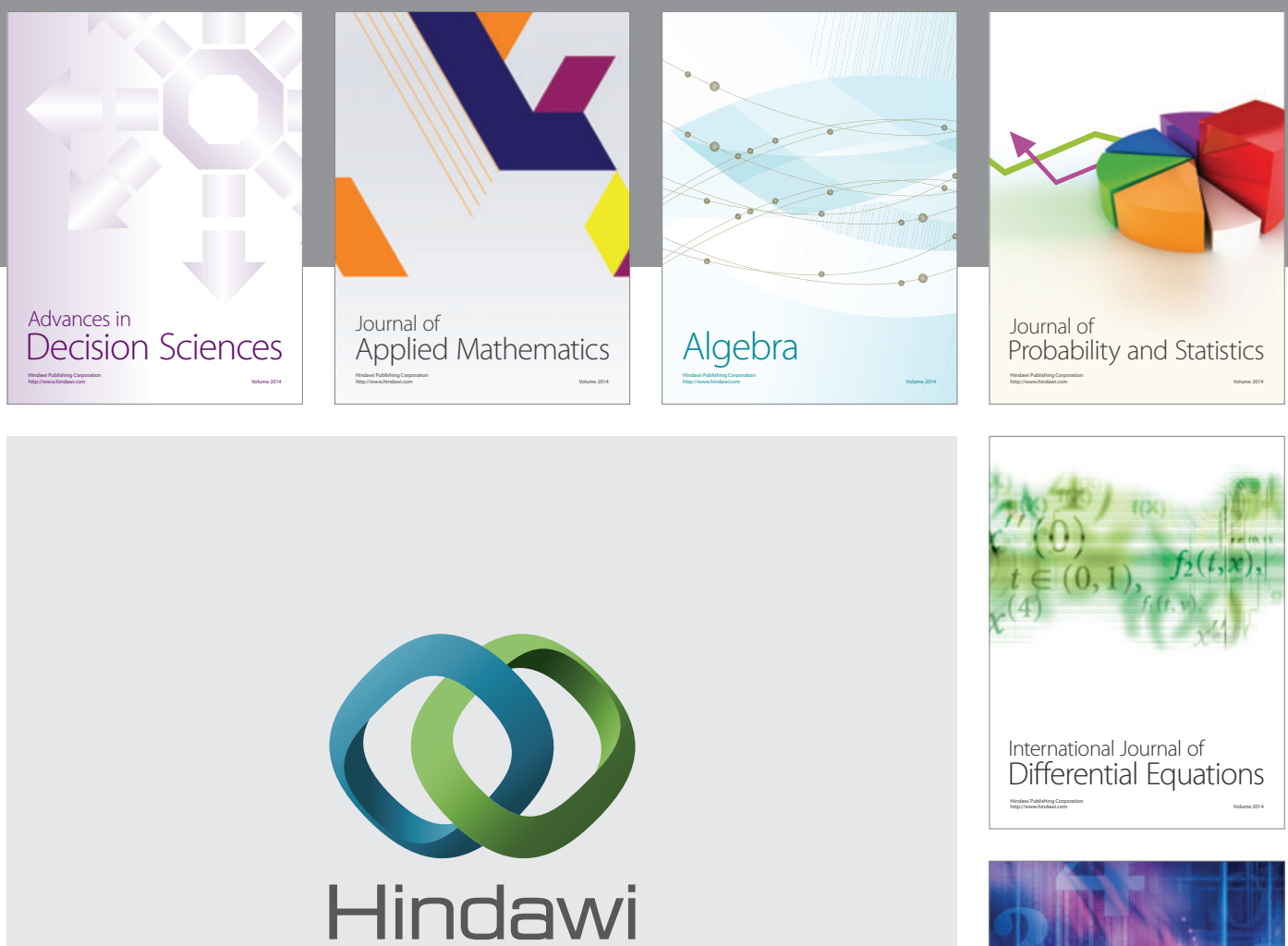

Submit your manuscripts at http://www.hindawi.com
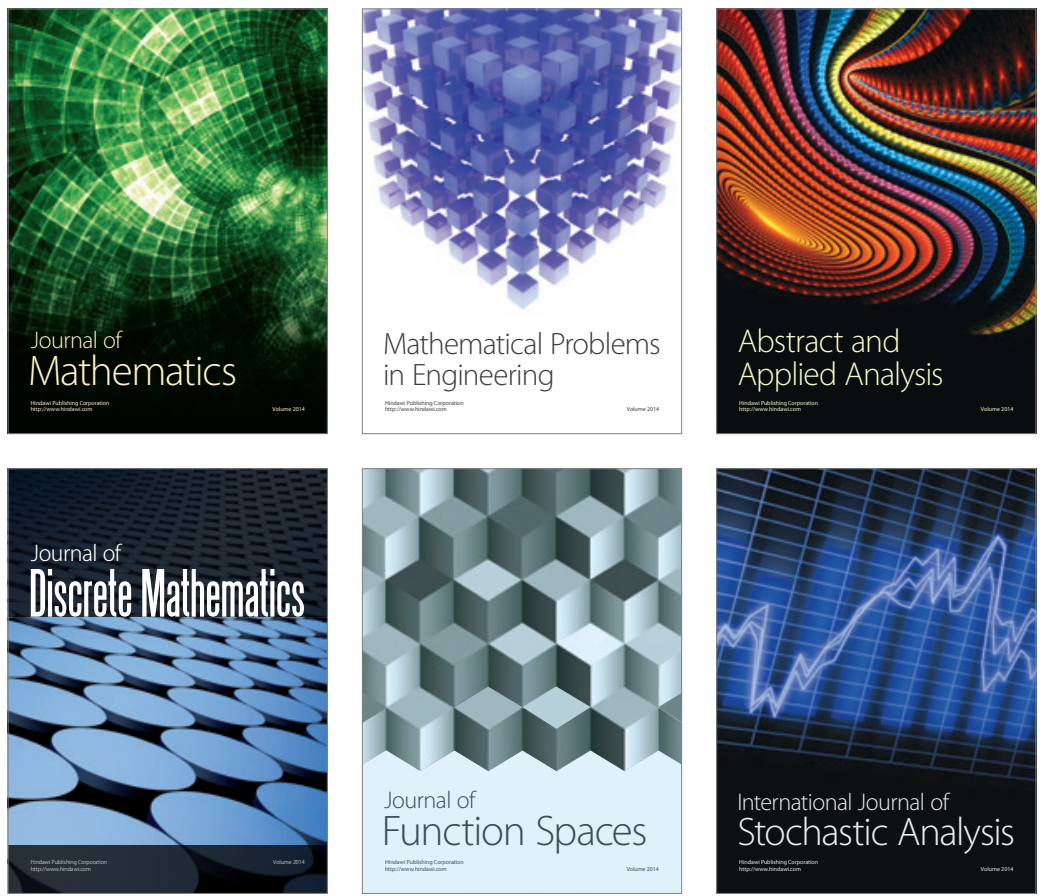

Journal of

Function Spaces

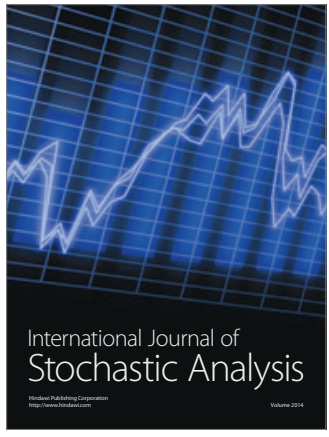

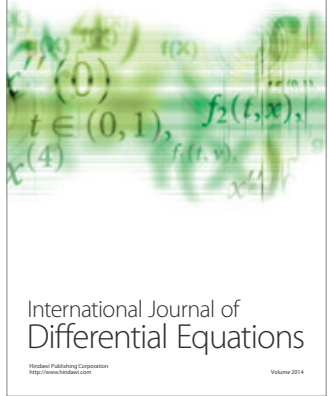
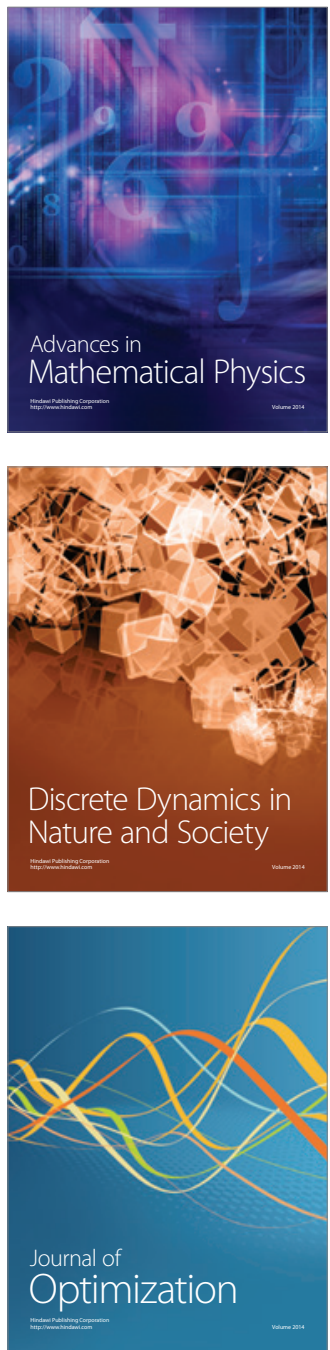